Monitoring the

Nation's Health

\title{
Oral Health Status and
} Access to Oral Health Care for U.S. Adults Aged 18-64: National Health Interview Survey, 2008 


\section{Copyright information}

All material appearing in this report is in the public domain and may be reproduced or copied without permission; citation as to source, however, is appreciated.

\section{Suggested citation}

Bloom B, Simile CM, Adams PF, Cohen RA. Oral health status and access to oral health care for U.S. adults aged 18-64: National Health Interview Survey, 2008. National Center for Health Statistics. Vital Health Stat 10(253). 2012.

\section{Library of Congress Cataloging-in-Publication Data}

Oral health status and access to oral health care for U.S. adults aged 18-64 : National Health Interview Survey, 2008.

p. ; cm.- (Vital and health statistics. Series 10, Data from the National Health Interview Survey ; no. 253) (DHHS publication ; no. (PHS)2012-1581) "July 2012."

Includes bibliographical references and index.

ISBN 978-0-8406-0653-2 (alk. paper)-ISBN 0-8406-0653-2 (alk. paper)

I. National Health Interview Survey (U.S.) II. National Center for Health

Statistics (U.S.) III. Series: Vital and health statistics. Series 10, Data from the

National Health Survey ; no. 253. IV. Series: DHHS publication ; no.

(PHS)2012-1581.

[DNLM: 1. Oral Health—statistics \& numerical data-United States. 2.

Adult-United States. 3. Dental Health Services-utilization-United States. 4.

Dental Health Surveys-United States. 5. Health Services Accessibility-

statistics \& numerical data-United States. 6. Health Status-United States-

Statistics. 7. Tooth Diseases—epidemiology-United States. W2 A N148vj

no.253 2012]

$362.197600973-d c 23$

2012026100

For sale by the U.S. Government Printing Office

Superintendent of Documents

Mail Stop: SSOP

Washington, DC 20402-9328

Printed on acid-free paper. 


\section{Vital and Health Statistics}

Series 10, Number 253

\section{Oral Health Status and Access to Oral Health Care for U.S. Adults Aged 18-64: National Health Interview Survey, 2008}

Data From the National Health Interview Survey 


\section{National Center for Health Statistics}

Edward J. Sondik, Ph.D., Director

Jennifer H. Madans, Ph.D., Associate Director for Science

Division of Health Interview Statistics

Jane F. Gentleman, Ph.D., Director 


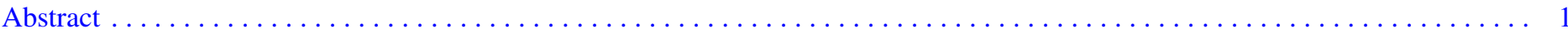

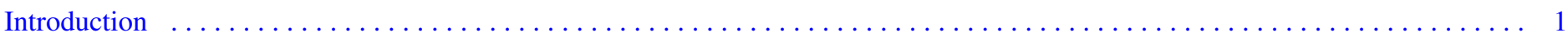

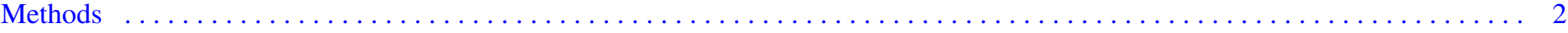

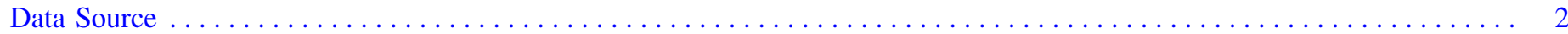

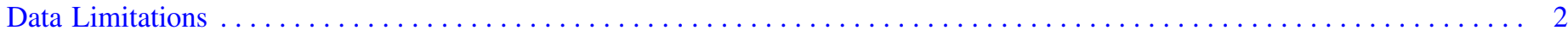

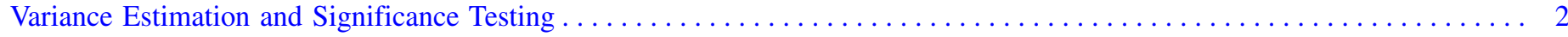

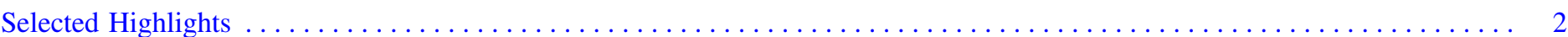

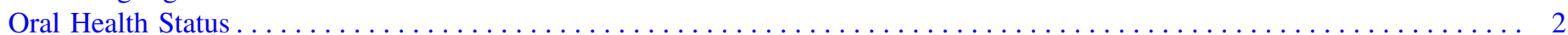

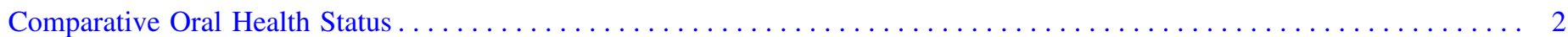

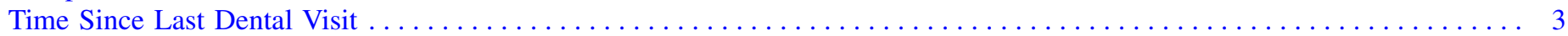

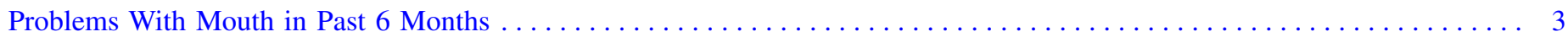

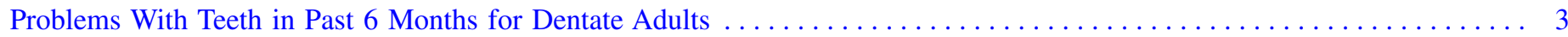

Reasons That Adults With an Oral Health Problem Did Not Visit a Dentist for Their Problem in Past 6 Months......... 3

Unmet Dental Need Due to Cost in Past 12 Months for Dentate Adults With and Without Oral Health Problems .......... 3

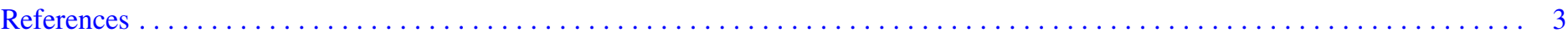

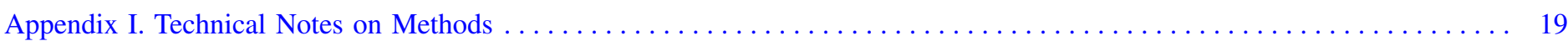

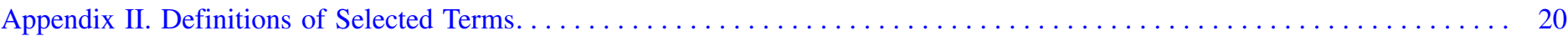

\section{Detailed Tables}

1. Percent distributions of oral health status among adults aged 18-64, by selected demographic and health characteristics:

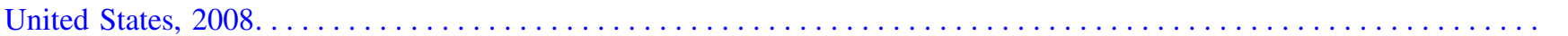

2. Percent distributions of comparative oral health status among adults aged 18-64, by selected demographic and health

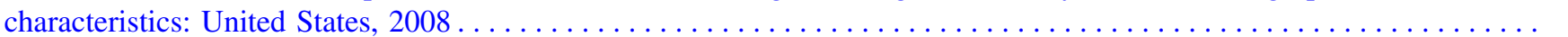

3. Percent distributions of length of time since last visit with a dentist or other dental health professional among adults aged 18-64, by selected demographic and health characteristics: United States, $2008 \ldots \ldots \ldots \ldots$

4. Percentages of adults aged 18-64 who had problems with mouth in the past 6 months, by selected demographic and health characteristics: United States, $2008 \ldots \ldots \ldots \ldots \ldots \ldots \ldots \ldots \ldots \ldots \ldots \ldots$

5. Percentages of dentate adults aged 18-64 who had problems with teeth in the past 6 months, by selected demographic and health characteristics: United States, 2008.

6. Percentages of adults aged 18-64 with a mouth or tooth problem who did not see a dentist or other dental health professional in the past 6 months, by reason and by selected demographic and health characteristics:

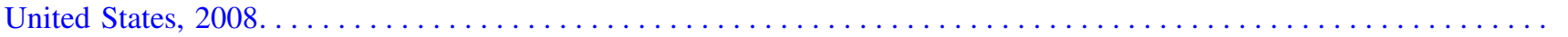

7. Percentages of dentate adults aged 18-64 who had an unmet dental need due to cost in the past 12 months, dentate adults with no mouth or teeth problems, with one or more mouth or teeth problems, and with toothache as a problem, by selected demographic and health characteristics: United States, 2008 . 


\section{Objectives}

This report presents statistics from the 2008 National Health Interview Survey (NHIS) on selected measures of oral health status and oral health care access for adults aged 18-64. Estimates are presented by sex, age, race and ethnicity, nativity, education, poverty status, health and dental insurance status, region, place of residence, dentition status, current smoking status, current drinking status, and diabetes status.

\section{Data Source}

NHIS is a multistage probability sample survey conducted annually by interviewers of the U.S. Census Bureau for the Centers for Disease Control and Prevention's National Center for Health Statistics, and is representative of the civilian noninstitutionalized population of the United States. Data are collected for all family members during face-toface interviews with adults present at the time of interview. Additional health information is obtained from one randomly selected adult. If the selected adult is physically or mentally incapable of responding for himself or herself, a proxy respondent is used.

\section{Selected Highlights}

Among adults aged 18-64, about three-quarters had very good or good oral health, $17 \%$ had fair oral health, and $7 \%$ had poor oral health. Adults with Medicaid were almost five times as likely as adults with private health insurance to have poor oral health. Adults with Medicaid (21\%) were almost twice as likely as adults overall (12\%) to not have had a dental visit in more than 5 years. Among adults aged 18-64, the main reason to forgo a dental visit for an oral health problem in the past 6 months was cost; $42 \%$ could not afford treatment or did not have insurance. Fear was the reason that 1 out of 10 adults did not visit the dentist for an oral health problem.

Keywords: dental care $\bullet$ dental problem • dental visit $\bullet$ unmet dental need

\section{Oral Health Status and Access to Oral Health Care for U.S. Adults Aged 18-64: National Health Interview Survey, 2008}

by Barbara Bloom, M.P.A.; Catherine M. Simile, Ph.D.; Patricia F. Adams; and Robin A. Cohen, Ph.D., Division of Health Interview Statistics

\section{Introduction}

In 2000, the U.S. Surgeon General issued a report (1) calling attention to the "silent epidemic" of dental and oral diseases suffered by millions of children and adults throughout the United States. The report emphasized the importance of oral health to overall health and noted that lifestyle behaviors that affect general health such as tobacco use, excessive alcohol use, and poor dietary choices also affect oral and craniofacial health. It observed that safe and effective measures do exist to prevent the most common dental diseases, dental caries and periodontal diseases, but it also underscored the disparities in oral health and access to oral health care within the U.S. population. The Surgeon General's report (1) and subsequent initiatives (2-4) emphasized the need for more information about the status of the U.S. population's oral health and the ability to access oral health care.

Based on the recommendations of the Surgeon General's report (1) and the National Call to Action to Promote Oral Health (2), the National Institute of Dental and Craniofacial Research (NIDCR) at the National Institutes of Health (NIH) sponsored a set of supplementary questions about oral health on the 2008 National Health Interview Survey (NHIS) (5). The purpose of the collaboration was twofold: 1) to develop and expand oral health outcome measures; and 2) to produce estimates for the U.S. population as a whole, and for racial and ethnic minorities, rural populations, the young, the elderly, and other subpopulations that could be used to study the impact of the initiatives to improve oral health.

This report uses data from the oral health supplement of the 2008 NHIS to provide prevalence estimates of oral health status; oral health status compared with others the same age; length of time since last dental visit; mouth problems, including bad breath, dry mouth, difficulty eating or chewing, jaw pain, and mouth sores; tooth problems, including toothache or sensitive teeth, stained or discolored teeth, broken or missing teeth, crooked teeth, bleeding gums, broken or missing fillings, and loose teeth not due to injury; reasons for not seeing a dentist for mouth or tooth problems; and unmet dental need due to cost for adults aged 18-64 living in households throughout the United States. Tables 1-7 are presented for various subgroups of the population, including those defined by sex, age, race and ethnicity, nativity, education, poverty status, health and dental insurance status, region, place of residence, dentition status, current smoking status, current drinking status, and diabetes status. Appendix I contains brief technical notes including information about the number of unknowns with respect to each health characteristic and the treatment of unknown values, and Appendix II contains the definitions of terms used in this report. 


\section{Methods}

\section{Data Source}

NHIS data are collected continuously throughout the year for the Centers for Disease Control and Prevention's National Center for Health Statistics (NCHS), by interviewers from the U.S. Census Bureau. NHIS collects information about a broad range of health topics for the civilian noninstitutionalized U.S. population. Interviews are conducted in respondents' homes, but followups to complete interviews may be conducted over the telephone. The Family component collects information about all family members from one responsible family member of the age of majority for a given state. The Sample Adult component collects additional data from one randomly selected adult (the "sample adult"). Self-response is required in the Sample Adult interview unless the designated respondent is physically or mentally incapable of responding for him- or herself, in which case a proxy may respond. If a child is present in the family, the Sample Child component collects additional data on one randomly selected child (the "sample child"). A knowledgeable adult in the family provides information about the sample child.

In addition, the 2008 NHIS included a set of supplementary oral health questions, cosponsored by NIDCR, which were added to the Sample Adult core. Estimates contained in this report are based on data collected in the 2008 Family core and the Sample Adult core. The report presents data on adults aged 18-64, the sample size for which is 17,337 . For further information about NHIS, including the questionnaire, visit the NHIS website at http://www. cdc.gov/nchs/nhis.htm.

\section{Data Limitations}

In this report, oral health is defined as health of the mouth and teeth. Oral health status is based on the sample adult's self-assessment of the condition of his or her mouth and teeth. No clinical measurements were taken; therefore, an individual's oral health status may differ from the evaluation of oral health status by a dental health professional. Respondents also may experience recall problems or have different cultural definitions of illness or health, either of which could result in inaccurate responses. Furthermore, as with all surveys, respondents may simply underreport characteristics or conditions that they consider undesirable. Thus, it is likely that some of the prevalence estimates presented in this report are conservative. Some underestimates due to item nonresponse and unknowns, which are excluded from the tables, may also exist.

Although many of the sociodemographic and health characteristics examined are likely to be correlated, this report is limited to highlighting the sociodemographic variations for each oral health status and oral health care access characteristic separately.

Some oral health problems may be associated with age. While the tables show estimates by various age groups, the prevalence estimates presented in the tables are not age adjusted in order to provide a measure of oral health burden. In addition, note that data presented in Tables 1-3, 5, and 6 were based on questions asked of dentate and edentulous persons, so both were included in these tables. Data presented in Tables 4 and 7 were based on questions asked only of dentate persons, so only dentate persons were included in these tables. Lastly, data presented in Table 6 were based on a question asked only of adults who indicated that they had a mouth or tooth problem as detailed in Tables 4 and 5.

\section{Variance Estimation and Significance Testing}

Estimates and associated standard errors shown in Tables 1-7 were generated using SUDAAN, a software package designed to handle complex sample designs such as that used by NHIS (6). Estimates were weighted to represent the U.S. civilian noninstitutionalized population.
Estimates with a relative standard error greater than $30 \%$ and less than or equal to $50 \%$ are identified with an asterisk preceding the estimate. Readers should exercise caution when interpreting these statistics. Estimates with a relative standard error more than 50\% are indicated with a dagger and are not shown.

Statistical tests performed to assess significance of differences between estimates were two-tailed $t$ tests at the 0.05 level with no adjustments for multiple comparisons. Terms such as "greater than" and "less than" indicate a statistically significant difference. Terms such as "similar" or "no difference" indicate that the statistics being compared were not significantly different. Lack of comment regarding the difference between any two statistics does not mean that the difference was tested and found to be not significant.

\section{Selected Highlights}

\section{Oral Health Status (Table 1)}

- Among adults aged 18-64, about three-quarters had very good or good oral health, $17 \%$ had fair oral health, and 7\% had poor oral health.

- Non-Hispanic white (37\%) and non-Hispanic Asian (37\%) adults were more likely than Hispanic $(25 \%)$ or non-Hispanic black (26\%) adults to have very good oral health.

- Adults with Medicaid (19\%) were almost five times as likely as adults with private health insurance (4\%) to have poor oral health.

\section{Comparative Oral Health Status (Table 2)}

- Level of education was positively associated with having better comparative oral health status; $20 \%$ of adults aged 18-64 with less than a high school diploma compared with $39 \%$ of adults with a bachelor's degree or higher had better oral health status than others the same age. 
- Adults with diabetes (29\%) were almost twice as likely as adults without diabetes $(16 \%)$ to have worse oral health status than others the same age.

- Adults in poor families $(28 \%)$ were more than twice as likely as adults in families that were not poor (13\%) to have worse oral health status than others the same age.

\section{Time Since Last Dental Visit (Table 3)}

- Overall, 6 out of 10 adults aged 18-64 last visited a dentist or other dental health professional within the past year.

- Adults with Medicaid (21\%) were almost twice as likely as adults overall $(12 \%)$ to not have had a dental visit in more than 5 years.

- Diabetic adults (19\%) were almost twice as likely as nondiabetic adults $(11 \%)$ to not have had a dental visit in more than 5 years.

\section{Problems With Mouth in Past 6 Months (Table 4)}

- Non-Hispanic Asian adults aged 18-64 experienced fewer mouth problems than Hispanic, nonHispanic white, or non-Hispanic black adults, including problems with bad breath, dry mouth, difficulty eating or chewing, or jaw pain.

- Former drinkers were about twice as likely as lifetime abstainers and one and one-half times as likely as current drinkers to have experienced bad breath, dry mouth, or difficulty eating or chewing.

- Edentulous adults (17\%) were almost twice as likely as dentate adults $(9 \%)$ to have had dry mouth.

\section{Problems With Teeth in Past 6 Months for Dentate Adults (Table 5)}

- Non-Hispanic Asian adults aged 18-64 were less likely than Hispanic, non-Hispanic white, or non-Hispanic black adults to have a variety of problems with their teeth, including stained or discolored teeth, broken or missing teeth, crooked teeth, bleeding gums, broken or missing fillings, or loose teeth not due to injury.

- Adults with Medicaid were more likely than uninsured adults to experience toothache or sensitive teeth, stained or discolored teeth, broken or missing teeth, broken or missing fillings, or loose teeth not due to injury.

- In general, lifetime abstainers from alcohol were less likely to have had problems with their teeth than current or former drinkers.

\section{Reasons That Adults With an Oral Health Problem Did Not Visit a Dentist for Their Problem in Past 6 Months (Table 6)}

- Among adults aged 18-64, the main reason to forgo a dental visit for an oral health problem in the past 6 months was cost; $42 \%$ could not afford treatment or did not have insurance.

- Adults with a bachelor's degree or higher $(33 \%)$ were almost twice as likely as those with less than a high school diploma (18\%) to forgo a dental visit for a problem because they thought it was not important.

- Fear was the reason that 1 out of 10 adults did not visit the dentist for an oral health problem.

\section{Unmet Dental Need Due to} Cost in Past 12 Months for Dentate Adults With and Without Oral Health Problems (Table 7)

- Overall, $16 \%$ of dentate adults aged 18-64 had an unmet dental need due to cost in the past 12 months.

- Among dentate adults with one or more mouth or teeth problems, more than one-half of those who were uninsured had unmet dental need due to cost. One-third of those with Medicaid had unmet dental need compared with almost one-quarter of those with private health insurance but no dental coverage, and more than one-tenth of those with private health insurance with dental coverage.

\section{References}

1. U.S. Department of Health and Human Services. Oral health in America: A report of the Surgeon General. Rockville, MD: National Institute of Dental and Craniofacial Research. 2000.

2. U.S. Department of Health and Human Services. National call to action to promote oral health. Rockville, MD: National Institute of Dental and Craniofacial Research. NIH Publication No. 03-5303. 2003.

3. U.S. Department of Health and Human Services. Healthy people 2010. Oral health objectives. Available from: http://www.healthypeople.gov/2020/ topicsobjectives2020/objectives list.aspx?topicId=32 [Accessed 6/20/12].

4. U.S. Department of Health and Human Services. Promoting and enhancing the oral health of the public: HHS Oral Health Initiative 2010. Available from: http://www.hrsa.gov/publichealth/ clinical/oralhealth/hhsinitiative.pdf [Accessed 6/20/12].

5. National Center for Health Statistics. 2008 National Health Interview Survey (NHIS) public use data release. NHIS survey description. Available from: ftp://ftp.cdc.gov/pub/health_statistics/ nchs/dataset_documentation/nhis/2008/ srvydesc.pdf. 2009.

6. RTI International. SUDAAN, Release 10.0 [computer software]. Research Triangle Park, NC: RTI International. 2008. Available from: http://www. rti.org/SUDAAN/.

7. Schenker N, Raghunathan TE, Chiu PL, et al. Multiple imputation of family income and personal earnings in the National Health Interview Survey: Methods and examples. National Center for Health Statistics. 2008. Available from: http://www.cdc.gov/nchs/data/ nhis/tecdoc.pdf.

8. DeNavas-Walt C, Proctor BD, Smith J. U.S. Census Bureau. Income, poverty, and health insurance coverage in the United States: 2007. Current Population Reports, P60-235. Washington, DC: U.S. Government Printing Office. 2008. 
Page $4 \square$ Series 10, No. 253

Available from: http://www.census.gov/ prod/2008pubs/p60-235.pdf.

9. Office of Management and Budget. Revisions to the standards for the classification of federal data on race and ethnicity. Fed Regist 62(210):58782-90. 1997.

10. Centers for Medicare \& Medicaid Services. Dental care. Available from: http://www.medicaid.gov/MedicaidCHIP-Program-Information/By-Topics/ Benefits/Dental-Care.html [Accessed 6/21/12].

11. Bloom B, Simpson G, Cohen RA, Parsons PE. 1997 Access to health care part 2: Working-age adults. National Center for Health Statistics. Vital Health Stat 10(197). 1997. 
Table 1. Percent distributions of oral health status among adults aged 18-64, by selected demographic and health characteristics: United States, 2008

\begin{tabular}{|c|c|c|c|c|c|}
\hline \multirow[b]{2}{*}{ Selected characteristic } & \multicolumn{5}{|c|}{ Oral health status ${ }^{1}$} \\
\hline & Total & $\begin{array}{l}\text { Very } \\
\text { good }\end{array}$ & Good & Fair & Poor \\
\hline & \multicolumn{5}{|c|}{ Percent distribution ${ }^{2}$ (standard error) } \\
\hline 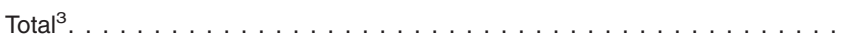 & 100.0 & $33.8(0.53)$ & $41.7(0.50)$ & $17.3(0.38)$ & $7.1(0.27)$ \\
\hline \multicolumn{6}{|l|}{ Sex } \\
\hline 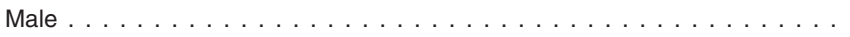 & 100.0 & $31.6(0.71)$ & $43.3(0.72)$ & $18.2(0.56)$ & $6.9(0.37)$ \\
\hline 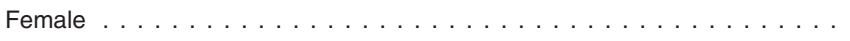 & 100.0 & $36.0(0.69)$ & $40.2(0.65)$ & $16.5(0.47)$ & $7.3(0.36)$ \\
\hline \multicolumn{6}{|l|}{ Age in years } \\
\hline 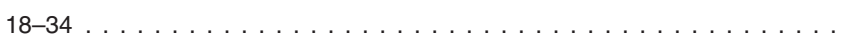 & 100.0 & $37.5(0.84)$ & $41.5(0.84)$ & $16.2(0.63)$ & $4.9(0.36)$ \\
\hline 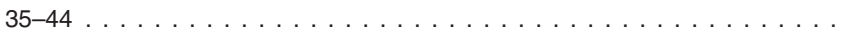 & 100.0 & $32.5(0.94)$ & $43.1(0.94)$ & $16.9(0.73)$ & $7.5(0.56)$ \\
\hline 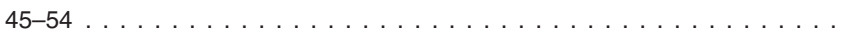 & 100.0 & $32.3(0.94)$ & $40.7(0.93)$ & $18.1(0.75)$ & $9.0(0.56)$ \\
\hline 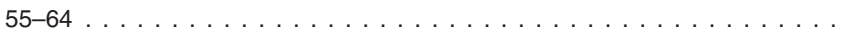 & 100.0 & $30.0(0.97)$ & $41.9(1.03)$ & $19.2(0.82)$ & $8.9(0.58)$ \\
\hline \multicolumn{6}{|l|}{ Race/ethnicity ${ }^{4}$} \\
\hline Hispanic or Latino . . . . . . . . . . . . . . . . . . . . . . . . . & 100.0 & $25.2(1.03)$ & $44.4(1.12)$ & $23.2(0.98)$ & $7.2(0.59)$ \\
\hline \multicolumn{6}{|l|}{ Non-Hispanic . . . . . . . . . . . . . . . . . . . . . . . . . . . } \\
\hline 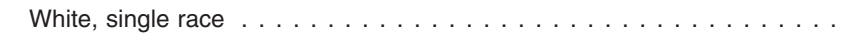 & 100.0 & $36.9(0.67)$ & $40.4(0.64)$ & $15.5(0.47)$ & $7.1(0.35)$ \\
\hline 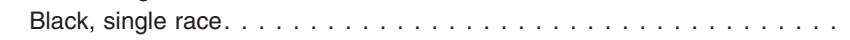 & 100.0 & $26.3(1.11)$ & $44.3(1.34)$ & $21.1(1.04)$ & $8.3(0.62)$ \\
\hline 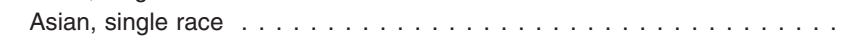 & 100.0 & $36.5(1.93)$ & $44.9(1.84)$ & $14.5(1.20)$ & $4.2(0.71)$ \\
\hline \multicolumn{6}{|l|}{ Nativity ${ }^{5}$} \\
\hline 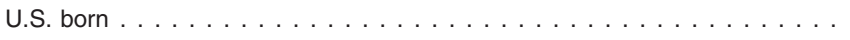 & 100.0 & $34.7(0.58)$ & $41.1(0.56)$ & $16.9(0.42)$ & $7.4(0.30)$ \\
\hline 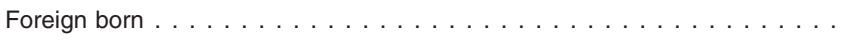 & 100.0 & $29.6(1.05)$ & $45.0(1.10)$ & $19.5(0.87)$ & $5.9(0.50)$ \\
\hline \multicolumn{6}{|l|}{ Education ${ }^{6}$} \\
\hline Less than a high school diploma $\ldots \ldots \ldots \ldots \ldots \ldots \ldots \ldots$ & 100.0 & $17.1(1.15)$ & $39.1(1.27)$ & $28.7(1.25)$ & $15.1(0.98)$ \\
\hline High school diploma or GED ${ }^{7} \ldots \ldots \ldots \ldots \ldots \ldots \ldots$ & 100.0 & $23.6(0.88)$ & $42.8(1.00)$ & $22.0(0.86)$ & $11.5(0.64)$ \\
\hline 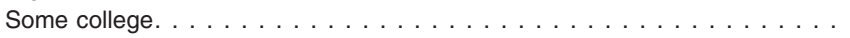 & 100.0 & $32.6(0.84)$ & $42.0(0.91)$ & $18.0(0.68)$ & $7.3(0.47)$ \\
\hline Bachelor's degree or higher . . . . . . . . . . . . . . . . . . & 100.0 & $46.1(0.92)$ & $41.9(0.91)$ & $9.8(0.52)$ & $2.1(0.27)$ \\
\hline \multicolumn{6}{|l|}{ Poverty status ${ }^{8}$} \\
\hline 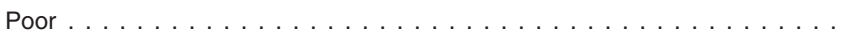 & 100.0 & $24.0(1.35)$ & $36.7(1.20)$ & $24.6(1.16)$ & $14.7(0.95)$ \\
\hline 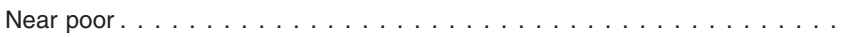 & 100.0 & $23.0(1.10)$ & $41.2(1.14)$ & $23.5(0.98)$ & $12.3(0.81)$ \\
\hline 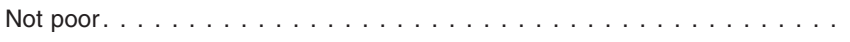 & 100.0 & $38.1(0.61)$ & $42.7(0.62)$ & $14.6(0.42)$ & $4.6(0.25)$ \\
\hline \multicolumn{6}{|l|}{ Health and dental insurance status ${ }^{9}$} \\
\hline Private health insurance $\ldots \ldots \ldots \ldots \ldots \ldots \ldots \ldots \ldots$ & 100.0 & $39.5(0.60)$ & $42.7(0.63)$ & $14.0(0.43)$ & $3.8(0.24)$ \\
\hline Dental coverage from single-service plan only $\ldots \ldots \ldots \ldots \ldots \ldots$ & 100.0 & $40.2(1.11)$ & $42.5(1.11)$ & $13.5(0.84)$ & $3.8(0.41)$ \\
\hline Dental coverage from comprehensive plan only $\ldots \ldots \ldots \ldots \ldots$ & 100.0 & $40.5(1.03)$ & $41.9(1.02)$ & $13.7(0.73)$ & $3.9(0.44)$ \\
\hline Dental coverage from both single-service and comprehensive plans . . . . & 100.0 & $37.9(1.57)$ & $46.9(1.64)$ & $11.7(0.99)$ & $3.5(0.59)$ \\
\hline 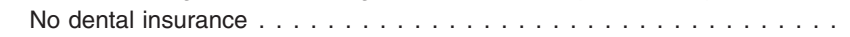 & 100.0 & $37.9(1.10)$ & $41.6(1.14)$ & $16.4(0.93)$ & $4.0(0.47)$ \\
\hline 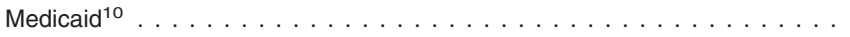 & 100.0 & $19.5(1.34)$ & $36.6(1.52)$ & $25.3(1.33)$ & $18.7(1.26)$ \\
\hline 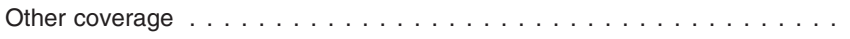 & 100.0 & $25.0(1.97)$ & $39.6(2.14)$ & $21.7(1.88)$ & $13.7(1.43)$ \\
\hline 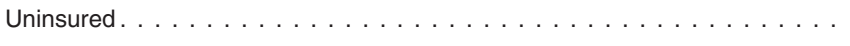 & 100.0 & $22.1(1.00)$ & $40.8(1.05)$ & $24.8(0.92)$ & $12.3(0.73)$ \\
\hline \multicolumn{6}{|l|}{ Region } \\
\hline 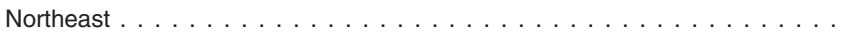 & 100.0 & $34.7(1.27)$ & $42.5(1.27)$ & $16.2(0.93)$ & $6.5(0.58)$ \\
\hline Midwest . . . . . . . . . . . . . . . . . . . . . . . . & 100.0 & $35.0(1.31)$ & $42.2(1.13)$ & $16.1(0.80)$ & $6.7(0.58)$ \\
\hline 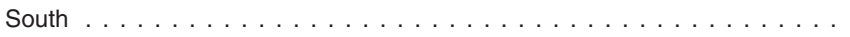 & 100.0 & $32.8(0.79)$ & $41.0(0.75)$ & $18.3(0.66)$ & $7.9(0.45)$ \\
\hline 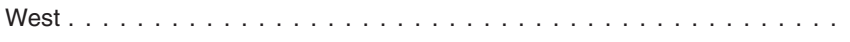 & 100.0 & $33.6(0.97)$ & $41.8(1.02)$ & $17.8(0.72)$ & $6.9(0.54)$ \\
\hline \multicolumn{6}{|l|}{ Place of residence } \\
\hline 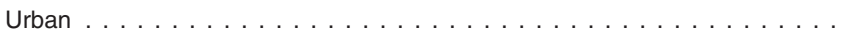 & 100.0 & $34.5(0.57)$ & $41.9(0.54)$ & $16.8(0.41)$ & $6.8(0.29)$ \\
\hline 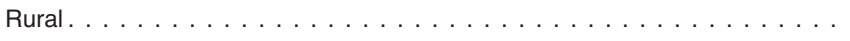 & 100.0 & $31.2(1.24)$ & $41.2(1.16)$ & $19.3(0.92)$ & $8.4(0.57)$ \\
\hline \multicolumn{6}{|l|}{ Dentition status } \\
\hline 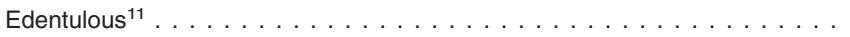 & 100.0 & $28.5(2.07)$ & $47.7(2.22)$ & $14.7(1.48)$ & $9.1(1.21)$ \\
\hline 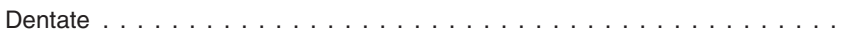 & 100.0 & $34.1(0.53)$ & $41.4(0.50)$ & $17.4(0.39)$ & $7.0(0.27)$ \\
\hline
\end{tabular}

See footnotes at end of table. 
Table 1. Percent distributions of oral health status among adults aged 18-64, by selected demographic and health characteristics: United States, 2008-Con.

\begin{tabular}{|c|c|c|c|c|c|}
\hline \multirow[b]{2}{*}{ Selected characteristic } & \multicolumn{5}{|c|}{ Oral health status ${ }^{1}$} \\
\hline & Total & $\begin{array}{l}\text { Very } \\
\text { good }\end{array}$ & Good & Fair & Poor \\
\hline Current smoking status ${ }^{12}$ & \multicolumn{5}{|c|}{ Percent distribution ${ }^{2}$ (standard error) } \\
\hline 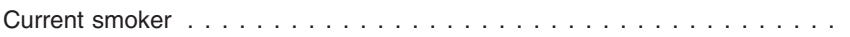 & 100.0 & $21.2(0.88)$ & $38.6(0.98)$ & $24.7(0.87)$ & $15.5(0.74)$ \\
\hline 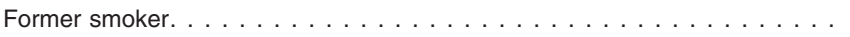 & 100.0 & $33.3(1.06)$ & $41.2(1.09)$ & $17.9(0.79)$ & $7.7(0.59)$ \\
\hline 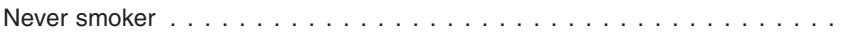 & 100.0 & $38.9(0.64)$ & $43.0(0.62)$ & $14.3(0.43)$ & $3.7(0.23)$ \\
\hline \multicolumn{6}{|l|}{ Current drinking status ${ }^{13}$} \\
\hline 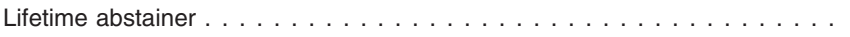 & 100.0 & $36.4(1.10)$ & $41.7(1.11)$ & $15.7(0.75)$ & $6.2(0.52)$ \\
\hline 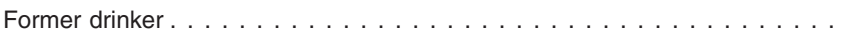 & 100.0 & $23.0(1.16)$ & $42.6(1.37)$ & $21.9(1.07)$ & $12.6(0.87)$ \\
\hline Current light or infrequent drinker $\ldots \ldots \ldots \ldots \ldots \ldots$ & 100.0 & $35.1(0.73)$ & $41.8(0.69)$ & $17.0(0.55)$ & $6.1(0.35)$ \\
\hline Current moderate or heavier drinker. . . . . . . . . . . . . . . & 100.0 & $34.9(1.00)$ & $40.9(1.06)$ & $17.0(0.79)$ & $7.2(0.57)$ \\
\hline \multicolumn{6}{|l|}{ Diabetes status ${ }^{14}$} \\
\hline 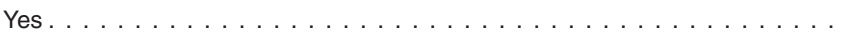 & 100.0 & $18.9(1.50)$ & $40.5(1.88)$ & $25.9(1.61)$ & $14.7(1.33)$ \\
\hline 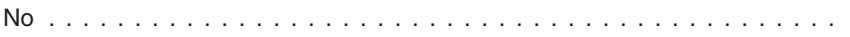 & 100.0 & $34.8(0.53)$ & $41.8(0.52)$ & $16.7(0.39)$ & $6.6(0.26)$ \\
\hline
\end{tabular}

1Based on the question: "How would you describe the condition of your mouth and teeth? Would you say very good, good, fair or poor?"

${ }^{2}$ Unknowns for the columns were not included in the denominators when calculating percentages. Percentages may not add to totals due to rounding.

${ }^{3}$ Includes other races and Hispanic origin not shown separately and persons with unknown nativity, education, health and dental insurance status, dentition status, current smoking status, current drinking status, and diabetes status.

${ }^{4}$ Persons of Hispanic or Latino origin may be of any race or combination of races. Non-Hispanic or Latino persons of a single race other than groups shown or of multiple races are not shown separately due to small sample sizes.

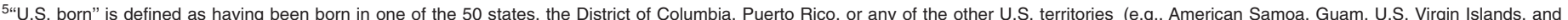

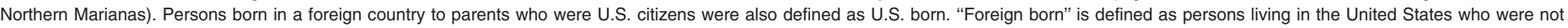
U.S. citizens at birth. All naturalized citizens, legal permanent residents, illegal aliens, and persons on long-term temporary visas (such as students or guest workers) also fall in this category. ${ }^{6}$ Shown only for persons aged 25 and over.

${ }^{7}$ GED is General Educational Development high school equivalency diploma.

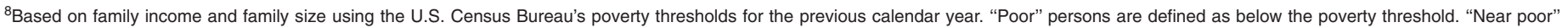
persons have incomes of $100 \%$ to less than $200 \%$ of the poverty threshold. "Not poor" persons have incomes that are $200 \%$ of the poverty threshold or greater. Because of the different income questions used in 2007 and beyond, poverty ratio estimates may not be comparable with those from earlier years.

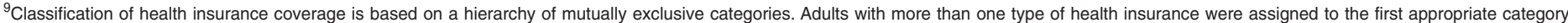

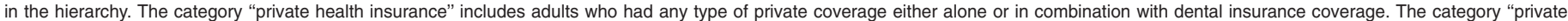

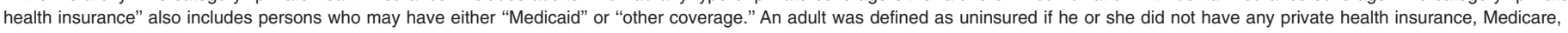

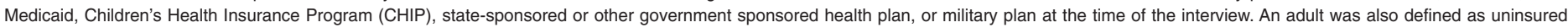

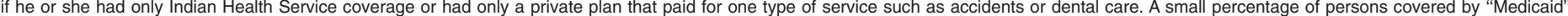

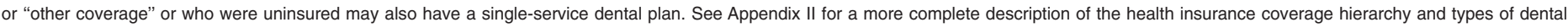
insurance coverage.

${ }^{10}$ Includes adults covered by CHIP or other state-sponsored health plans.

${ }^{11}$ Refers to persons who have lost all of their upper and lower natural teeth.

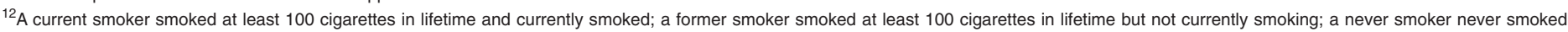
at all or smoked less than 100 cigarettes in lifetime.

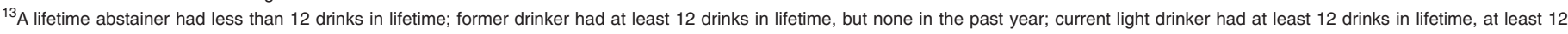

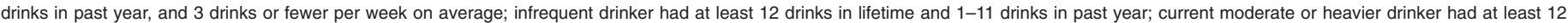
drinks in lifetime, at least 12 drinks in past year, and drank more than 3 drinks per week, on average.

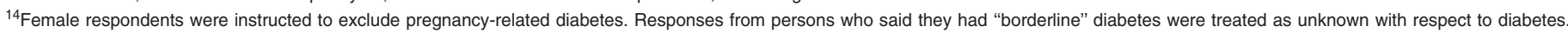
NOTE: Estimates are based on household interviews of a sample of the resident civilian noninstitutionalized population.

SOURCE: CDC/NCHS, National Health Interview Survey, 2008. 
Table 2. Percent distributions of comparative oral health status among adults aged 18-64, by selected demographic and health characteristics: United States, 2008

\begin{tabular}{|c|c|c|c|c|}
\hline \multirow[b]{2}{*}{ Selected characteristic } & \multicolumn{4}{|c|}{ Comparative oral health status ${ }^{1}$} \\
\hline & Total & $\begin{array}{l}\text { Better than } \\
\text { others the } \\
\text { same age }\end{array}$ & $\begin{array}{l}\text { Same as } \\
\text { others the } \\
\text { same age }\end{array}$ & $\begin{array}{l}\text { Not as good } \\
\text { as others the } \\
\text { same age }\end{array}$ \\
\hline & \multicolumn{4}{|c|}{ Percent distribution ${ }^{2}$ (standard error) } \\
\hline$\ldots \ldots \ldots \ldots \ldots \ldots \ldots \ldots \ldots$ & 100.0 & $29.9(0.47)$ & $53.5(0.51)$ & $16.5(0.39)$ \\
\hline \multicolumn{5}{|l|}{ Sex } \\
\hline 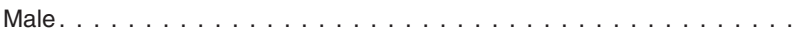 & 100.0 & $29.2(0.68)$ & $54.4(0.73)$ & $16.4(0.55)$ \\
\hline 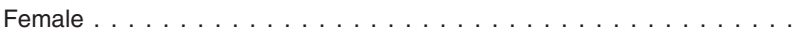 & 100.0 & $30.7(0.58)$ & $52.7(0.65)$ & $16.6(0.51)$ \\
\hline \multicolumn{5}{|l|}{ Age in years } \\
\hline 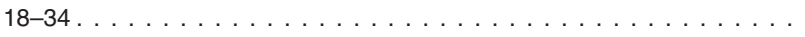 & 100.0 & $28.1(0.78)$ & $57.0(0.84)$ & $14.9(0.59)$ \\
\hline 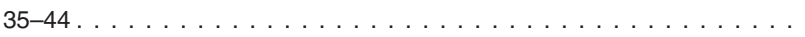 & 100.0 & $28.3(0.92)$ & $54.8(0.99)$ & $16.9(0.74)$ \\
\hline 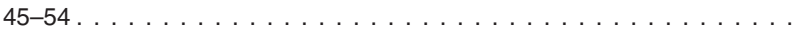 & 100.0 & $30.6(0.90)$ & $50.9(1.02)$ & $18.5(0.79)$ \\
\hline 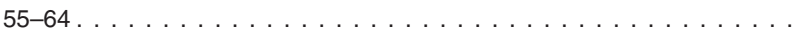 & 100.0 & $35.1(1.04)$ & $48.0(1.13)$ & $16.9(0.84)$ \\
\hline \multicolumn{5}{|l|}{ Race/ethnicity ${ }^{4}$} \\
\hline Hispanic or Latino . . . . . . . . . . . . . . . . . . . . . . . . . . & 100.0 & $25.9(1.07)$ & $57.1(1.17)$ & $16.9(0.91)$ \\
\hline \multicolumn{5}{|l|}{ 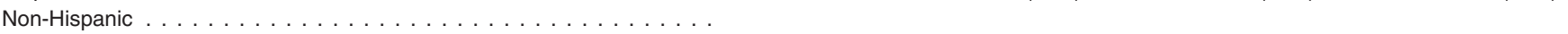 } \\
\hline 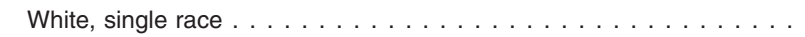 & 100.0 & $30.8(0.59)$ & $52.5(0.65)$ & $16.6(0.51)$ \\
\hline 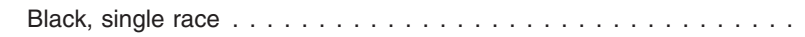 & 100.0 & $30.4(1.13)$ & $52.2(1.20)$ & $17.4(0.91)$ \\
\hline 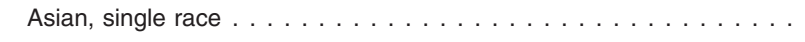 & 100.0 & $28.7(1.83)$ & $58.5(1.82)$ & $12.8(1.19)$ \\
\hline \multicolumn{5}{|l|}{ Nativity ${ }^{5}$} \\
\hline U.S. born . . . . . . . . . . . . . . . . . . . . . . & 100.0 & $30.3(0.52)$ & $52.8(0.56)$ & $16.9(0.44)$ \\
\hline 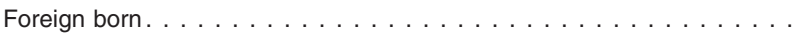 & 100.0 & $27.9(0.97)$ & $57.4(1.08)$ & $14.7(0.79)$ \\
\hline \multicolumn{5}{|l|}{ Education $^{6}$} \\
\hline Less than a high school diploma $\ldots \ldots \ldots \ldots \ldots \ldots$ & 100.0 & $19.5(1.10)$ & $51.4(1.40)$ & $29.0(1.31)$ \\
\hline High school diploma or $\mathrm{GED}^{7} \ldots \ldots \ldots \ldots \ldots \ldots \ldots \ldots$ & 100.0 & $25.5(0.90)$ & $51.6(1.00)$ & $22.9(0.94)$ \\
\hline 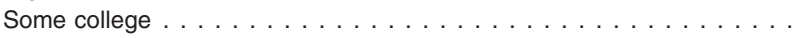 & 100.0 & $30.2(0.85)$ & $52.9(0.98)$ & $16.9(0.66)$ \\
\hline Bachelor's degree or higher $\ldots \ldots \ldots \ldots \ldots \ldots \ldots$ & 100.0 & $38.5(0.93)$ & $53.1(0.96)$ & $8.4(0.50)$ \\
\hline \multicolumn{5}{|l|}{ Poverty status ${ }^{8}$} \\
\hline 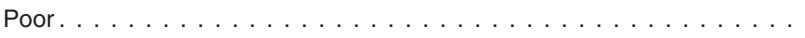 & 100.0 & $21.9(1.09)$ & $50.5(1.30)$ & $27.7(1.19)$ \\
\hline 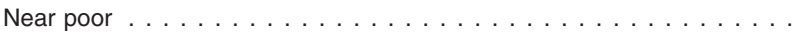 & 100.0 & $22.6(0.96)$ & $53.5(1.15)$ & $23.9(1.05)$ \\
\hline 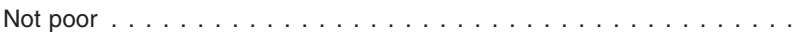 & 100.0 & $33.0(0.58)$ & $54.1(0.61)$ & $12.9(0.40)$ \\
\hline \multicolumn{5}{|l|}{ Health and dental insurance status ${ }^{9}$} \\
\hline Private health insurance. . . . . . . . . . . . . . . . . & 100.0 & $33.7(0.59)$ & $54.4(0.63)$ & $11.8(0.39)$ \\
\hline Dental coverage from single-service plan only. . . . . . . . . . . & 100.0 & $35.3(1.19)$ & $52.8(1.22)$ & $11.9(0.73)$ \\
\hline Dental coverage from comprehensive plan only. . . . . . . . . . . & 100.0 & $33.5(1.02)$ & $55.1(1.06)$ & $11.4(0.67)$ \\
\hline Dental coverage from both single-service and comprehensive plans . . & 100.0 & $34.4(1.47)$ & $54.3(1.61)$ & $11.3(1.03)$ \\
\hline 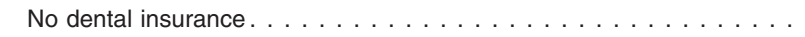 & 100.0 & $31.8(1.05)$ & $55.3(1.17)$ & $12.9(0.86)$ \\
\hline 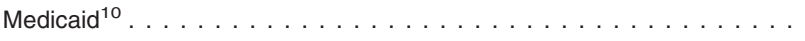 & 100.0 & $20.3(1.30)$ & $46.5(1.59)$ & $33.2(1.53)$ \\
\hline 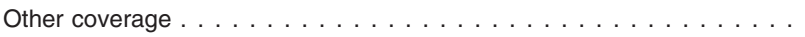 & 100.0 & $27.2(1.95)$ & $47.2(2.22)$ & $25.6(1.91)$ \\
\hline 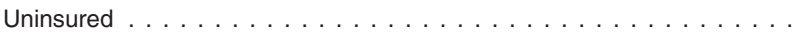 & 100.0 & $21.3(0.85)$ & $54.4(1.06)$ & $24.3(0.92)$ \\
\hline \multicolumn{5}{|l|}{ Region } \\
\hline 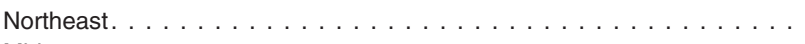 & 100.0 & $27.2(1.15)$ & $57.0(1.23)$ & $15.8(0.86)$ \\
\hline 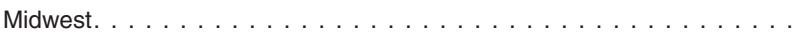 & 100.0 & $31.7(1.11)$ & $53.2(1.16)$ & $15.1(0.74)$ \\
\hline 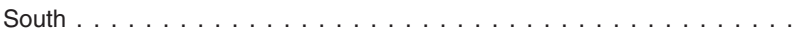 & 100.0 & $30.6(0.70)$ & $51.5(0.74)$ & $17.9(0.69)$ \\
\hline West. . . . . . . . . . . . . . . . . . . . . & 100.0 & $29.1(0.94)$ & $54.6(1.07)$ & $16.2(0.81)$ \\
\hline \multicolumn{5}{|l|}{ Place of residence } \\
\hline 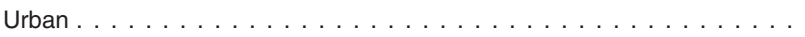 & 100.0 & $30.2(0.53)$ & $53.8(0.57)$ & $16.0(0.43)$ \\
\hline 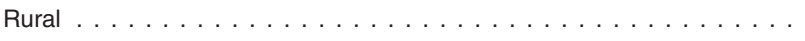 & 100.0 & $28.8(0.99)$ & $52.4(1.05)$ & $18.7(0.89)$ \\
\hline \multicolumn{5}{|l|}{ Dentition status } \\
\hline Edentulous $^{11} \ldots \ldots \ldots \ldots \ldots \ldots \ldots$ & 100.0 & $20.0(1.80)$ & $52.1(2.12)$ & $27.9(1.95)$ \\
\hline 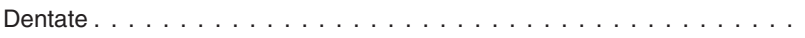 & 100.0 & $30.5(0.49)$ & $53.6(0.52)$ & $15.9(0.39)$ \\
\hline
\end{tabular}

See footnotes at end of table. 
Table 2. Percent distributions of comparative oral health status among adults aged 18-64, by selected demographic and health characteristics: United States, 2008-Con.

\begin{tabular}{|c|c|c|c|c|}
\hline \multirow[b]{2}{*}{ Selected characteristic } & \multicolumn{4}{|c|}{ Comparative oral health status ${ }^{1}$} \\
\hline & Total & $\begin{array}{l}\text { Better than } \\
\text { others the } \\
\text { same age }\end{array}$ & $\begin{array}{l}\text { Same as } \\
\text { others the } \\
\text { same age }\end{array}$ & $\begin{array}{c}\text { Not as good } \\
\text { as others the } \\
\text { same age }\end{array}$ \\
\hline Current smoking status ${ }^{12}$ & \multicolumn{4}{|c|}{ Percent distribution ${ }^{2}$ (standard error) } \\
\hline 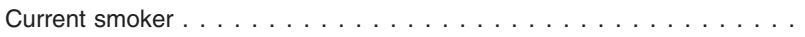 & 100.0 & $20.8(0.81)$ & $48.3(1.04)$ & $30.9(0.99)$ \\
\hline Former smoker $\ldots \ldots \ldots \ldots \ldots \ldots \ldots \ldots \ldots \ldots \ldots \ldots$ & 100.0 & $32.6(1.01)$ & $50.4(1.08)$ & $17.0(0.85)$ \\
\hline Never smoker . . . . . . . . . . . . . . . . . . . . & 100.0 & $32.7(0.59)$ & $56.4(0.63)$ & $10.9(0.38)$ \\
\hline \multicolumn{5}{|l|}{ Current drinking status ${ }^{13}$} \\
\hline Lifetime abstainer. . . . . . . . . . . . . . . . . . . & 100.0 & $27.1(0.94)$ & $58.9(1.08)$ & $14.0(0.74)$ \\
\hline 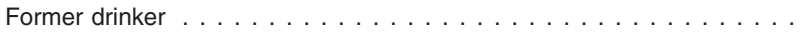 & 100.0 & $25.3(1.14)$ & $51.0(1.36)$ & $23.7(1.14)$ \\
\hline Current light or infrequent drinker $\ldots \ldots \ldots \ldots \ldots$ & 100.0 & $31.4(0.67)$ & $52.8(0.70)$ & $15.8(0.53)$ \\
\hline Current moderate or heavier drinker $\ldots \ldots \ldots \ldots \ldots \ldots$ & 100.0 & $32.4(1.01)$ & $51.1(1.07)$ & $16.6(0.81)$ \\
\hline \multicolumn{5}{|l|}{ Diabetes status ${ }^{14}$} \\
\hline 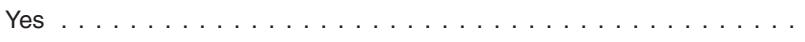 & 100.0 & $24.3(1.52)$ & $47.1(1.76)$ & $28.6(1.68)$ \\
\hline 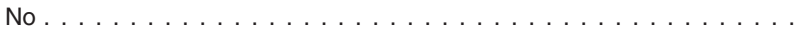 & 100.0 & $30.3(0.48)$ & $54.0(0.52)$ & $15.7(0.39)$ \\
\hline
\end{tabular}

1Based on the question: "Would you say the condition of your mouth and teeth is better than, the same as or not as good as other people your age?"

${ }^{2}$ Unknowns for the columns were not included in the denominators when calculating percentages. Percentages may not add to total due to rounding.

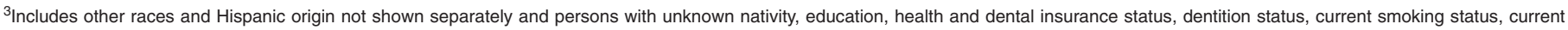
drinking status, and diabetes status.

${ }^{4}$ Persons of Hispanic or Latino origin may be of any race or combination of races. Non-Hispanic or Latino persons of a single race other than groups shown or of multiple races are not shown separately due to small sample sizes.

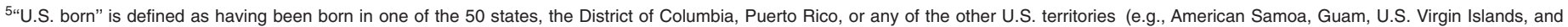

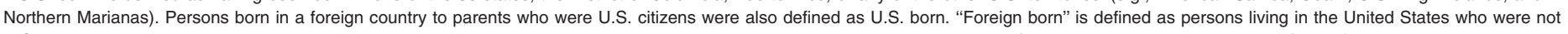
U.S. citizens at birth. All naturalized citizens, legal permanent residents, illegal aliens, and persons on long-term temporary visas (such as students or guest workers) also fall in this category. ${ }^{6}$ Shown only for persons aged 25 and over.

${ }^{7}$ GED is General Educational Development high school equivalency diploma.

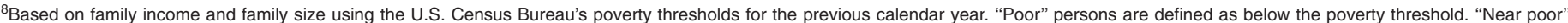
persons have incomes of $100 \%$ to less than $200 \%$ of the poverty threshold. "Not poor" persons have incomes that are $200 \%$ of the poverty threshold or greater. Because of the different income questions used in 2007 and beyond, poverty ratio estimates may not be comparable with those from earlier years.

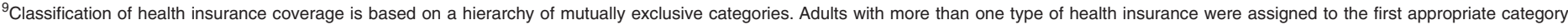

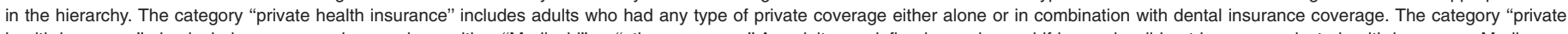

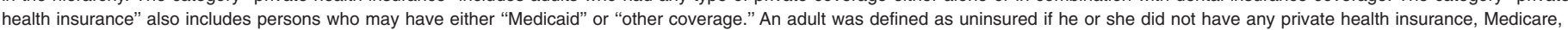

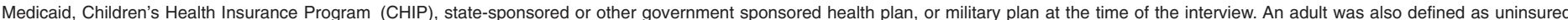

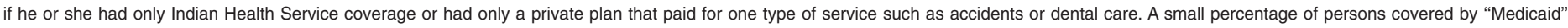

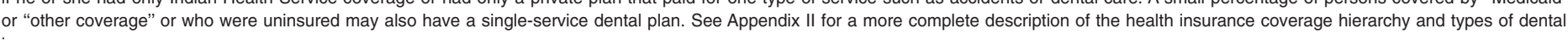
insurance coverage.

${ }^{10}$ Includes adults covered by CHIP or other state-sponsored health plans.

${ }^{11}$ Refers to persons who have lost all of their upper and lower natural teeth.

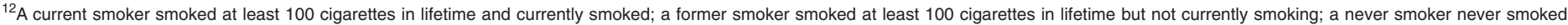
at all or smoked less than 100 cigarettes in lifetime.

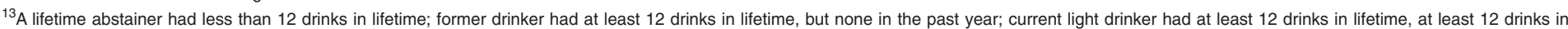

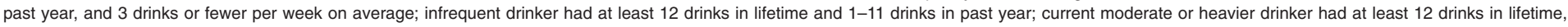
at least 12 drinks in past year, and drank more than 3 drinks per week, on average.

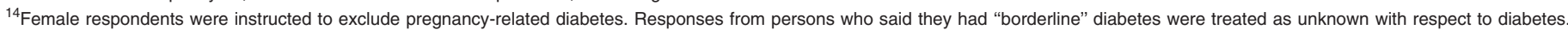

NOTE: Estimates are based on household interviews of a sample of the resident civilian noninstitutionalized population.

SOURCE: CDC/NCHS, National Health Interview Survey, 2008. 
Table 3. Percent distributions of length of time since last visit with a dentist or other dental health professional among adults aged 18-64, by selected demographic and health characteristics: United States, 2008

\begin{tabular}{|c|c|c|c|c|c|c|c|}
\hline \multirow[b]{2}{*}{ Selected characteristic } & \multicolumn{7}{|c|}{ Length of time since last visit ${ }^{1}$} \\
\hline & Total & $\begin{array}{l}6 \text { months } \\
\text { or less }\end{array}$ & $\begin{array}{c}\text { More than } \\
6 \text { months, but } \\
\text { not more than } \\
1 \text { year ago }\end{array}$ & $\begin{array}{c}\text { More than } \\
1 \text { year, but } \\
\text { not more than } \\
2 \text { years ago }\end{array}$ & $\begin{array}{c}\text { More than } \\
2 \text { years, but } \\
\text { not more than } \\
5 \text { years ago }\end{array}$ & $\begin{array}{l}\text { More than } \\
5 \text { years, } \\
\text { excluding } \\
\text { "Never" }\end{array}$ & Never \\
\hline & \multicolumn{7}{|c|}{ Percent distribution ${ }^{2}$ (standard error) } \\
\hline$\ldots \ldots \ldots \ldots \ldots \ldots \ldots$ & 100.0 & $42.4(0.53)$ & $18.0(0.38)$ & $13.7(0.33)$ & $12.8(0.34)$ & $11.7(0.34)$ & $1.4(0.12)$ \\
\hline \multicolumn{8}{|l|}{ Sex } \\
\hline Male. . . . . . . . . . . . . . . . & 100.0 & $38.9(0.72)$ & $17.5(0.57)$ & $15.1(0.50)$ & $13.3(0.49)$ & $13.6(0.50)$ & $1.6(0.17)$ \\
\hline Female $\ldots \ldots \ldots \ldots \ldots \ldots \ldots \ldots$ & 100.0 & $45.9(0.68)$ & $18.5(0.49)$ & $12.4(0.41)$ & $12.2(0.42)$ & $9.9(0.40)$ & $1.1(0.15)$ \\
\hline \multicolumn{8}{|l|}{ Age in years } \\
\hline 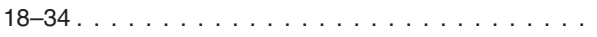 & 100.0 & $35.6(0.81)$ & $20.2(0.68)$ & $16.6(0.59)$ & $14.6(0.58)$ & $10.7(0.50)$ & $2.2(0.25)$ \\
\hline 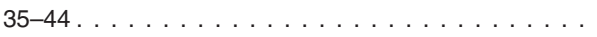 & 100.0 & $42.7(0.99)$ & $18.6(0.79)$ & $13.3(0.63)$ & $12.3(0.59)$ & $11.9(0.67)$ & $1.3(0.19)$ \\
\hline 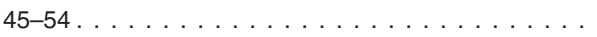 & 100.0 & $48.0(1.01)$ & $16.2(0.69)$ & $11.4(0.60)$ & $12.0(0.65)$ & $11.8(0.64)$ & $0.6(0.14)$ \\
\hline 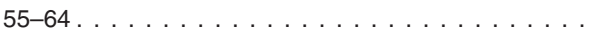 & 100.0 & $49.0(1.04)$ & $15.1(0.72)$ & $11.2(0.68)$ & $10.5(0.66)$ & $13.5(0.69)$ & $0.7(0.20)$ \\
\hline \multicolumn{8}{|l|}{ Race/ethnicity ${ }^{4}$} \\
\hline Hispanic or Latino . . . . . . . . . . . . . . . . . . . . & 100.0 & $28.2(1.07)$ & $17.5(0.87)$ & $17.1(0.96)$ & $16.0(0.82)$ & $16.8(0.86)$ & $4.5(0.53)$ \\
\hline \multicolumn{8}{|l|}{ 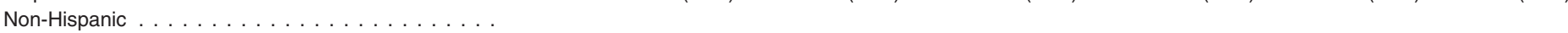 } \\
\hline White, single race $\ldots \ldots \ldots \ldots \ldots$ & 100.0 & $47.8(0.68)$ & $17.4(0.47)$ & $12.3(0.39)$ & $11.3(0.41)$ & $10.7(0.42)$ & $0.6(0.10)$ \\
\hline Black, single race $\ldots \ldots \ldots \ldots \ldots \ldots$ & 100.0 & $31.5(1.19)$ & $21.3(1.00)$ & $16.2(0.91)$ & $17.2(0.98)$ & $12.2(0.78)$ & $1.6(0.31)$ \\
\hline Asian, single race $\ldots \ldots \ldots \ldots \ldots \ldots$ & 100.0 & $40.8(1.88)$ & $19.8(1.47)$ & $14.9(1.34)$ & $12.7(1.27)$ & $9.5(1.16)$ & $2.3(0.48)$ \\
\hline \multicolumn{8}{|l|}{ Nativity $^{5}$} \\
\hline U.S. born. . . . . . . . . . . . . . . . . . . . & 100.0 & $44.3(0.59)$ & $17.9(0.41)$ & $13.4(0.35)$ & $12.3(0.37)$ & $11.4(0.37)$ & $0.6(0.08)$ \\
\hline Foreign born . . . . . . . . . . . . . . . . & 100.0 & $33.2(1.07)$ & $18.5(0.83)$ & $15.2(0.87)$ & $14.9(0.78)$ & $13.1(0.73)$ & $5.1(0.52)$ \\
\hline \multicolumn{8}{|l|}{ Education $^{6}$} \\
\hline Less than a high school diploma . . . . . . . . . . . . . & 100.0 & $23.5(1.21)$ & $14.3(0.97)$ & $14.6(1.04)$ & $18.8(1.09)$ & $24.9(1.23)$ & $4.0(0.54)$ \\
\hline High school diploma or $\mathrm{GED}^{7} \ldots \ldots \ldots \ldots$ & 100.0 & $35.3(0.97)$ & $15.8(0.70)$ & $14.5(0.66)$ & $15.6(0.74)$ & $17.8(0.78)$ & $1.0(0.18)$ \\
\hline Some college . . . . . . . . . . . . . . . . . . & 100.0 & $43.6(0.91)$ & $19.7(0.74)$ & $14.0(0.62)$ & $12.1(0.60)$ & $10.0(0.51)$ & $0.6(0.14)$ \\
\hline Bachelor's degree or higher . . . . . . . . . . . . . & 100.0 & $59.5(0.87)$ & $18.2(0.68)$ & $10.2(0.52)$ & $7.3(0.45)$ & $4.3(0.37)$ & $0.5(0.12)$ \\
\hline \multicolumn{8}{|l|}{ Poverty status ${ }^{8}$} \\
\hline Poor. . . . . . . . . . . . . . . & 100.0 & $25.4(1.34)$ & $15.9(0.99)$ & $15.7(1.00)$ & $17.0(1.00)$ & $22.5(1.20)$ & $3.5(0.52)$ \\
\hline Near poor . . . . . . . . . . . . . . . & 100.0 & $25.7(1.06)$ & $15.2(0.81)$ & $16.7(0.81)$ & $19.5(0.96)$ & $20.3(0.99)$ & $2.6(0.40)$ \\
\hline Not poor $\ldots \ldots \ldots \ldots \ldots \ldots \ldots$ & 100.0 & $49.3(0.62)$ & $19.0(0.46)$ & $12.7(0.38)$ & $10.5(0.37)$ & $7.9(0.31)$ & $0.7(0.10)$ \\
\hline \multicolumn{8}{|l|}{ Health and dental insurance status ${ }^{9}$} \\
\hline Private health insurance. . . . . . . . . . . . . & 100.0 & $51.3(0.62)$ & $19.6(0.47)$ & $12.3(0.38)$ & $9.6(0.35)$ & $6.6(0.28)$ & $0.5(0.08)$ \\
\hline Dental coverage from single-service plan only. . . . & 100.0 & $60.4(1.06)$ & $18.1(0.82)$ & $10.4(0.66)$ & $6.5(0.53)$ & $4.3(0.47)$ & ${ }^{*} 0.2(0.07)$ \\
\hline Dental coverage from comprehensive plan only. . . & 100.0 & $51.2(1.03)$ & $20.3(0.79)$ & $12.6(0.65)$ & $9.1(0.59)$ & $6.2(0.53)$ & $0.7(0.17)$ \\
\hline $\begin{array}{l}\text { Dental coverage from both single-service and } \\
\text { comprehensive plans } \ldots \ldots \ldots \ldots \ldots \ldots\end{array}$ & 100.0 & $53.1(1.57)$ & $20.1(1.29)$ & $11.4(1.02)$ & $9.1(0.88)$ & $6.0(0.72)$ & ${ }^{*} 0.3(0.16)$ \\
\hline No dental insurance . . . . . . . . . . . . . . . & 100.0 & $41.8(1.18)$ & $20.5(0.97)$ & $14.2(0.76)$ & $13.5(0.85)$ & $9.3(0.68)$ & $0.7(0.18)$ \\
\hline Medicaid $^{10} \ldots \ldots \ldots \ldots \ldots \ldots \ldots$ & 100.0 & $30.3(1.55)$ & $15.4(1.08)$ & $14.6(1.09)$ & $17.3(1.16)$ & $20.8(1.33)$ & $1.5(0.34)$ \\
\hline Other coverage $\ldots \ldots \ldots \ldots \ldots \ldots \ldots$ & 100.0 & $37.3(2.13)$ & $15.6(1.55)$ & $16.0(1.63)$ & $13.4(1.50)$ & $17.3(1.67)$ & $\dagger$ \\
\hline Uninsured $\ldots \ldots \ldots \ldots \ldots \ldots \ldots$ & 100.0 & $17.9(0.84)$ & $13.9(0.69)$ & $18.0(0.91)$ & $21.5(0.94)$ & $24.4(1.00)$ & $4.5(0.47)$ \\
\hline \multicolumn{8}{|l|}{ Region } \\
\hline Northeast. . . . . . . . . . . . . . . . . . . . . . . & 100.0 & $48.7(1.37)$ & $19.6(0.99)$ & $10.7(0.75)$ & $10.7(0.83)$ & $9.2(0.73)$ & $1.1(0.21)$ \\
\hline Midwest. . . . . . . . . . . . . . . . . . & 100.0 & $45.4(1.09)$ & $17.9(0.85)$ & $14.0(0.64)$ & $10.9(0.62)$ & $11.2(0.79)$ & $0.6(0.11)$ \\
\hline South . . . . . . . . . . . . . . . . . & 100.0 & $37.7(0.84)$ & $17.5(0.58)$ & $14.1(0.58)$ & $15.2(0.61)$ & $13.7(0.56)$ & $1.7(0.20)$ \\
\hline West. . . . . . . . . . . . . . . . . & 100.0 & $42.1(1.05)$ & $17.7(0.72)$ & $14.9(0.67)$ & $12.5(0.63)$ & $10.9(0.61)$ & $2.0(0.34)$ \\
\hline \multicolumn{8}{|l|}{ Place of residence } \\
\hline Urban . . . . . . . . . . . . . . . . & 100.0 & $42.7(0.59)$ & $18.2(0.42)$ & $14.0(0.37)$ & $12.7(0.36)$ & $10.9(0.34)$ & $1.5(0.13)$ \\
\hline Rural $\ldots \ldots \ldots \ldots \ldots \ldots \ldots \ldots \ldots$ & 100.0 & $41.2(1.21)$ & $17.4(0.85)$ & $12.7(0.67)$ & $13.1(0.77)$ & $14.8(0.95)$ & $1.0(0.26)$ \\
\hline
\end{tabular}


Table 3. Percent distributions of length of time since last visit with a dentist or other dental health professional among adults aged 18-64, by selected demographic and health characteristics: United States, 2008-Con.

\begin{tabular}{|c|c|c|c|c|c|c|c|}
\hline \multirow[b]{2}{*}{ Selected characteristic } & \multicolumn{7}{|c|}{ Length of time since last visit ${ }^{1}$} \\
\hline & Total & $\begin{array}{c}6 \text { months } \\
\text { or less }\end{array}$ & $\begin{array}{l}\text { More than } \\
6 \text { months, but } \\
\text { not more than } \\
1 \text { year ago }\end{array}$ & $\begin{array}{c}\text { More than } \\
1 \text { year, but } \\
\text { not more than } \\
2 \text { years ago }\end{array}$ & $\begin{array}{c}\text { More than } \\
2 \text { years, but } \\
\text { not more than } \\
5 \text { years ago }\end{array}$ & $\begin{array}{l}\text { More than } \\
5 \text { years, } \\
\text { excluding } \\
\text { "Never" }\end{array}$ & Never \\
\hline Dentition status & \multicolumn{7}{|c|}{ Percent distribution ${ }^{2}$ (standard error) } \\
\hline Edentulous ${ }^{11}$. & 100.0 & $24.8(1.85)$ & $12.0(1.34)$ & $11.9(1.32)$ & $16.7(1.48)$ & $32.9(1.98)$ & $1.6(0.42)$ \\
\hline Dentate . . . . . . . . . . . . . . . . . . . & 100.0 & $43.4(0.54)$ & $18.3(0.39)$ & $13.8(0.34)$ & $12.6(0.34)$ & $10.6(0.32)$ & $1.4(0.12)$ \\
\hline \multicolumn{8}{|l|}{ Current smoking status ${ }^{12}$} \\
\hline Current smoker . . . . . . . . . . . . . . . & 100.0 & $32.9(0.93)$ & $15.8(0.72)$ & $14.1(0.66)$ & $16.9(0.77)$ & $19.3(0.80)$ & $1.1(0.18)$ \\
\hline Former smoker . . . . . . . . . . . . . . . . & 100.0 & $47.6(1.11)$ & $16.6(0.80)$ & $13.2(0.73)$ & $11.3(0.70)$ & $10.8(0.69)$ & $0.6(0.16)$ \\
\hline Never smoker . . . . . . . . . . . . . . . . . . . & 100.0 & $44.6(0.67)$ & $19.3(0.52)$ & $13.8(0.43)$ & $11.6(0.39)$ & $9.1(0.35)$ & $1.7(0.17)$ \\
\hline \multicolumn{8}{|l|}{ Current drinking status ${ }^{13}$} \\
\hline Lifetime abstainer. . . . . . . . . . . . . . . . . . & 100.0 & $36.0(1.10)$ & $19.3(0.84)$ & $15.4(0.83)$ & $13.9(0.79)$ & $11.9(0.67)$ & $3.5(0.42)$ \\
\hline Former drinker $\ldots \ldots \ldots \ldots \ldots \ldots \ldots$ & 100.0 & $36.3(1.33)$ & $15.1(0.94)$ & $14.7(0.90)$ & $15.1(0.95)$ & $18.2(1.03)$ & *0.6 (0.21) \\
\hline Current light or infrequent drinker $\ldots \ldots \ldots \ldots$ & 100.0 & $45.8(0.72)$ & $18.9(0.53)$ & $13.1(0.49)$ & $11.8(0.46)$ & $9.6(0.43)$ & $0.8(0.11)$ \\
\hline Current moderate or heavier drinker $\ldots \ldots \ldots \ldots$ & 100.0 & $44.7(1.06)$ & $16.6(0.78)$ & $12.9(0.68)$ & $12.3(0.68)$ & $12.4(0.74)$ & $1.0(0.20)$ \\
\hline \multicolumn{8}{|l|}{ Diabetes status ${ }^{14}$} \\
\hline 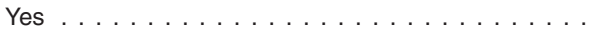 & 100.0 & $40.0(1.81)$ & $12.9(1.24)$ & $12.7(1.20)$ & $14.7(1.34)$ & $18.8(1.29)$ & *1.0 (0.38) \\
\hline 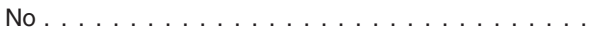 & 100.0 & $42.6(0.54)$ & $18.3(0.38)$ & $13.8(0.34)$ & $12.6(0.34)$ & $11.2(0.34)$ & $1.4(0.12)$ \\
\hline
\end{tabular}

* Estimate has a relative standard error of greater than $30 \%$ and less than or equal to $50 \%$ and should be used with caution as it does not meet standards of reliability or precision.

$\dagger$ Estimate has a relative standard error greater than $50 \%$ and is not shown.

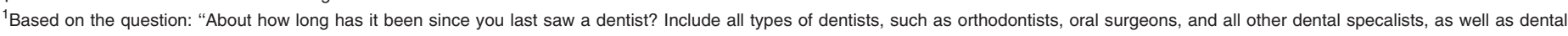
hygienists."

${ }^{2}$ Unknowns for the columns were not included in the denominators when calculating percentages. Percentages may not add to totals due to rounding

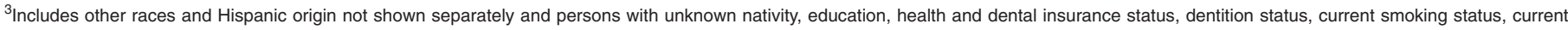
drinking status, and diabetes status.

${ }^{4}$ Persons of Hispanic or Latino origin may be of any race or combination of races. Non-Hispanic or Latino persons of a single race other than groups shown or of multiple races are not shown separately due to small sample sizes.

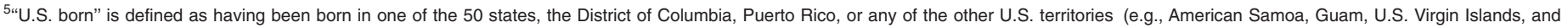

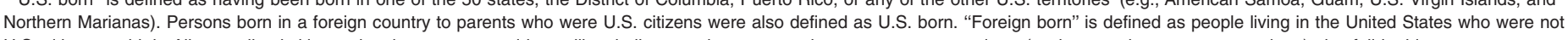
U.S. citizens at birth. All naturalized citizens, legal permanent residents, illegal aliens, and persons on long-term temporary visas (such as students or guest workers) also fall in this category.

${ }^{6}$ Shown only for persons aged 25 and over.

${ }^{7}$ GED is General Educational Development high school equivalency diploma.

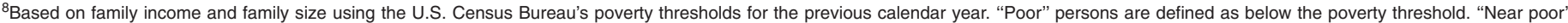
persons have incomes of $100 \%$ to less than $200 \%$ of the poverty threshold. "Not poor" persons have incomes that are $200 \%$ of the poverty threshold or greater. Because of the different income questions used in 2007 and beyond, poverty ratio estimates may not be comparable with those from earlier years.

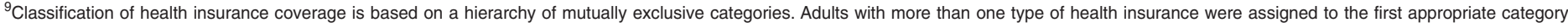

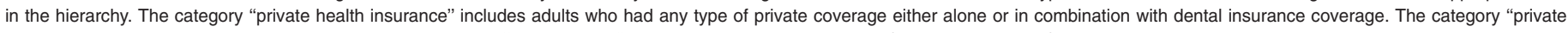

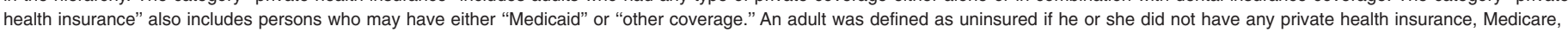

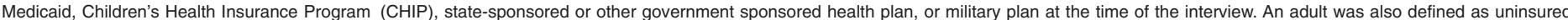

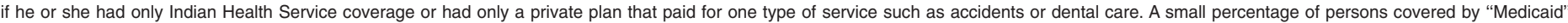

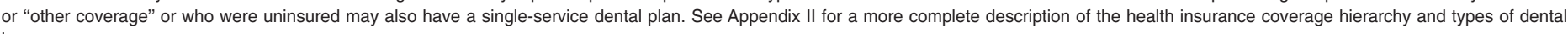
insurance coverage.

${ }^{10}$ Includes adults covered by CHIP or other state-sponsored health plans.

${ }^{11}$ Refers to persons who have lost all of their upper and lower natural teeth.

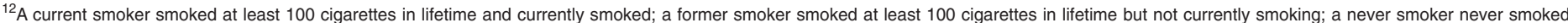
at all or smoked less than 100 cigarettes in lifetime.

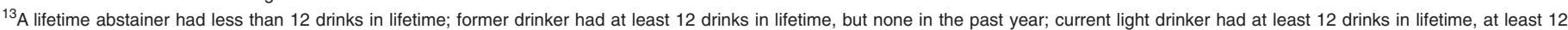

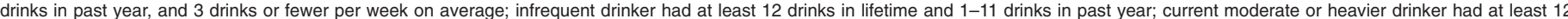
drinks in lifetime, at least 12 drinks in past year, and drank more than 3 drinks per week, on average.

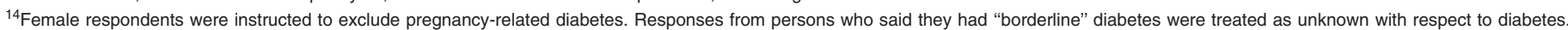

NOTE: Estimates are based on household interviews of a sample of the resident civilian noninstitutionalized population.

SOURCE: CDC/NCHS, National Health Interview Survey, 2008. 
Table 4. Percentages of adults aged 18-64 who had problems with mouth in the past 6 months, by selected demographic and health characteristics: United States, 2008

\begin{tabular}{|c|c|c|c|c|c|}
\hline \multirow[b]{2}{*}{ Selected characteristic } & \multicolumn{5}{|c|}{ Problem with mouth ${ }^{1}$} \\
\hline & Bad breath & Dry mouth & $\begin{array}{l}\text { Difficulty } \\
\text { eating or } \\
\text { chewing }\end{array}$ & Jaw pain & Mouth sores \\
\hline & \multicolumn{5}{|c|}{ Percent $^{2}$ (standard error) } \\
\hline Total $^{3} \ldots \ldots \ldots \ldots \ldots \ldots \ldots \ldots \ldots$ & $9.8(0.31)$ & $9.7(0.29)$ & $8.0(0.27)$ & $7.2(0.26)$ & $5.4(0.23)$ \\
\hline \multicolumn{6}{|l|}{ Sex } \\
\hline 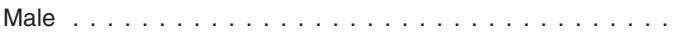 & $10.4(0.44)$ & $8.4(0.39)$ & $7.5(0.37)$ & $5.6(0.33)$ & $5.1(0.31)$ \\
\hline Female . . . . . . . . . . . . . . . . & $9.2(0.38)$ & $11.0(0.41)$ & $8.5(0.36)$ & $8.7(0.39)$ & $5.7(0.31)$ \\
\hline \multicolumn{6}{|l|}{ Age in years } \\
\hline 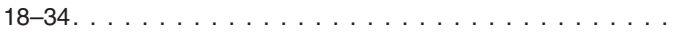 & $8.4(0.49)$ & $7.2(0.42)$ & $7.0(0.42)$ & $8.1(0.47)$ & $6.1(0.41)$ \\
\hline 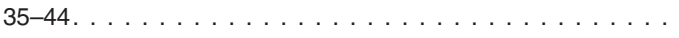 & $10.6(0.62)$ & $8.8(0.55)$ & $8.0(0.57)$ & $6.5(0.47)$ & $4.6(0.41)$ \\
\hline 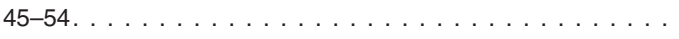 & $10.6(0.61)$ & $11.8(0.63)$ & $8.9(0.56)$ & $7.0(0.53)$ & $5.9(0.47)$ \\
\hline 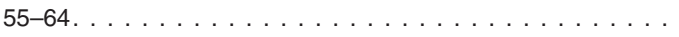 & $10.6(0.64)$ & $13.3(0.71)$ & $9.1(0.59)$ & $6.4(0.51)$ & $4.4(0.42)$ \\
\hline \multicolumn{6}{|l|}{ Race/ethnicity ${ }^{4}$} \\
\hline Hispanic or Latino . . . . . . . . . . . . . . . . . . . . . . & $9.7(0.68)$ & $9.1(0.63)$ & $7.7(0.59)$ & $6.1(0.56)$ & $4.8(0.48)$ \\
\hline \multicolumn{6}{|l|}{ Non-Hispanic . . . . . . . . . . . . . . . . . . . . . . } \\
\hline White, single race $\ldots \ldots \ldots \ldots \ldots \ldots \ldots$ & $10.5(0.41)$ & $10.2(0.37)$ & $8.1(0.34)$ & $7.8(0.35)$ & $6.0(0.30)$ \\
\hline Black, single race $\ldots \ldots \ldots \ldots \ldots \ldots \ldots \ldots$ & $7.9(0.57)$ & $9.1(0.68)$ & $8.4(0.68)$ & $6.2(0.60)$ & $2.4(0.39)$ \\
\hline Asian, single race $\ldots \ldots \ldots \ldots \ldots \ldots$ & $5.7(0.87)$ & $5.9(0.97)$ & $5.3(0.88)$ & $3.8(0.81)$ & $6.3(0.93)$ \\
\hline \multicolumn{6}{|l|}{ Nativity ${ }^{5}$} \\
\hline U.S. born. . . . . . . . . . . . . . . . . . . . . . & $10.3(0.35)$ & $10.2(0.33)$ & $8.3(0.30)$ & $7.7(0.30)$ & $5.6(0.26)$ \\
\hline Foreign born. . . . . . . . . . . . . . . . . . & $7.0(0.53)$ & $7.1(0.53)$ & $6.5(0.55)$ & $4.5(0.47)$ & $4.5(0.44)$ \\
\hline \multicolumn{6}{|l|}{ Education $^{6}$} \\
\hline Less than a high school diploma $\ldots \ldots \ldots \ldots \ldots$ & $15.1(0.98)$ & $16.0(1.03)$ & $13.8(0.90)$ & $8.7(0.76)$ & $6.0(0.67)$ \\
\hline High school diploma or $\mathrm{GED}^{7} \ldots \ldots \ldots \ldots \ldots$. . . . . . & $12.0(0.63)$ & $11.8(0.62)$ & $9.5(0.56)$ & $7.3(0.53)$ & $4.3(0.42)$ \\
\hline Some college . . . . . . . . . . . . . . . . . . & $9.9(0.56)$ & $10.9(0.59)$ & $8.9(0.53)$ & $8.1(0.49)$ & $5.8(0.45)$ \\
\hline Bachelor's degree or higher $\ldots \ldots \ldots \ldots \ldots$ & $6.7(0.43)$ & $6.3(0.44)$ & $4.6(0.38)$ & $5.3(0.41)$ & $5.0(0.39)$ \\
\hline \multicolumn{6}{|l|}{ Poverty status ${ }^{8}$} \\
\hline Poor. . . . . . . . . . . . . . . . . & $13.8(0.95)$ & $16.4(0.96)$ & $14.6(0.93)$ & $11.5(0.84)$ & $7.1(0.65)$ \\
\hline Near poor . . . . . . . . . . . . . . . . . & $13.3(0.80)$ & $13.1(0.77)$ & $11.6(0.70)$ & $9.1(0.72)$ & $5.7(0.54)$ \\
\hline Not poor $\ldots \ldots \ldots \ldots \ldots \ldots \ldots \ldots \ldots$ & $8.3(0.34)$ & $7.8(0.31)$ & $6.0(0.29)$ & $6.0(0.29)$ & $5.0(0.28)$ \\
\hline \multicolumn{6}{|l|}{ Health and dental insurance status ${ }^{9}$} \\
\hline Private health insurance $\ldots \ldots \ldots \ldots \ldots \ldots$ & $8.0(0.34)$ & $7.5(0.31)$ & $5.6(0.28)$ & $6.0(0.29)$ & $5.0(0.29)$ \\
\hline Dental coverage from single-service plan only $\ldots \ldots \ldots$ & $8.5(0.63)$ & $7.6(0.58)$ & $6.2(0.52)$ & $7.7(0.66)$ & $5.4(0.52)$ \\
\hline Dental coverage from comprehensive plan only $\ldots \ldots \ldots$ & $6.6(0.50)$ & $7.0(0.54)$ & $5.2(0.52)$ & $5.2(0.48)$ & $4.3(0.45)$ \\
\hline $\begin{array}{l}\text { Dental coverage from both single-service and } \\
\text { comprehensive plans } \ldots \ldots \ldots \ldots \ldots \ldots \ldots \ldots \ldots \ldots\end{array}$ & $8.8(0.91)$ & $7.4(0.76)$ & $5.4(0.67)$ & $5.0(0.61)$ & $4.9(0.69)$ \\
\hline No dental insurance. . . . . . . . . . . . . . . . . . . . & $8.6(0.68)$ & $7.9(0.59)$ & $5.8(0.53)$ & $5.6(0.57)$ & $5.4(0.60)$ \\
\hline Medicaid $^{10} \ldots \ldots \ldots \ldots \ldots \ldots \ldots \ldots \ldots \ldots$ & $15.8(1.19)$ & $19.2(1.27)$ & $16.9(1.12)$ & $10.7(1.00)$ & $6.0(0.74)$ \\
\hline Other coverage $\ldots \ldots \ldots \ldots \ldots \ldots \ldots \ldots \ldots$ & $15.9(1.66)$ & $20.4(1.75)$ & $16.8(1.70)$ & $11.4(1.53)$ & $6.1(1.13)$ \\
\hline Uninsured . . . . . . . . . . . . . . . . . . . . & $12.2(0.72)$ & $11.4(0.64)$ & $10.9(0.62)$ & $9.0(0.66)$ & $6.3(0.53)$ \\
\hline \multicolumn{6}{|l|}{ Region } \\
\hline 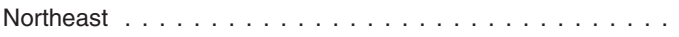 & $8.9(0.65)$ & $9.1(0.63)$ & $7.7(0.66)$ & $6.6(0.59)$ & $5.6(0.69)$ \\
\hline Midwest . . . . . . . . . . . . . . . . & $9.8(0.65)$ & $9.3(0.62)$ & $7.8(0.57)$ & $7.3(0.58)$ & $5.1(0.48)$ \\
\hline South . . . . . . . . . . . . . . . . . . . . & $9.7(0.53)$ & $9.5(0.48)$ & $7.8(0.41)$ & $7.4(0.45)$ & $5.2(0.36)$ \\
\hline 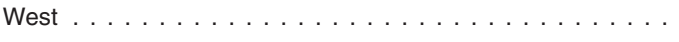 & $10.5(0.63)$ & $11.0(0.59)$ & $8.8(0.60)$ & $7.2(0.51)$ & $5.8(0.46)$ \\
\hline \multicolumn{6}{|l|}{ Place of residence } \\
\hline Urban. . . . . . . . . . . . . . . . . . . . & $9.3(0.33)$ & $9.6(0.31)$ & $7.8(0.29)$ & $7.0(0.28)$ & $5.4(0.25)$ \\
\hline Rural . . . . . . . . . . . . . . . . . . . & $11.5(0.79)$ & $10.2(0.65)$ & $9.0(0.62)$ & $7.8(0.63)$ & $5.6(0.57)$ \\
\hline
\end{tabular}

See footnotes at end of table. 
Table 4. Percentages of adults aged 18-64 who had problems with mouth in the past 6 months, by selected demographic and health characteristics: United States, 2008-Con.

\begin{tabular}{|c|c|c|c|c|c|}
\hline \multirow[b]{2}{*}{ Selected characteristic } & \multicolumn{5}{|c|}{ Problem with mouth ${ }^{1}$} \\
\hline & Bad breath & Dry mouth & $\begin{array}{l}\text { Difficulty } \\
\text { eating or } \\
\text { chewing }\end{array}$ & Jaw pain & Mouth sores \\
\hline Dentition status & \multicolumn{5}{|c|}{ Percent $^{2}$ (standard error) } \\
\hline Edentulous $^{11} \ldots \ldots \ldots \ldots \ldots \ldots \ldots \ldots$ & $9.5(1.31)$ & $17.2(1.55)$ & $12.8(1.38)$ & $8.1(1.21)$ & $8.7(1.53)$ \\
\hline Dentate. . . . . . . . . . . . . . . . . . & $9.8(0.32)$ & $9.3(0.29)$ & $7.8(0.27)$ & $7.1(0.27)$ & $5.2(0.22)$ \\
\hline \multicolumn{6}{|l|}{ Current smoking status ${ }^{12}$} \\
\hline Current smoker . . . . . . . . . . . . . . . . . & $15.0(0.73)$ & $15.5(0.70)$ & $14.0(0.66)$ & $10.4(0.59)$ & $5.7(0.52)$ \\
\hline Former smoker $\ldots \ldots \ldots \ldots \ldots \ldots \ldots \ldots$ & $10.0(0.68)$ & $10.9(0.68)$ & $8.3(0.60)$ & $7.8(0.60)$ & $5.5(0.47)$ \\
\hline Never smoker . . . . . . . . . . . . . . . . . . . . . & $7.7(0.34)$ & $7.2(0.31)$ & $5.6(0.31)$ & $5.8(0.33)$ & $5.3(0.30)$ \\
\hline \multicolumn{6}{|l|}{ Current drinking status ${ }^{13}$} \\
\hline Lifetime abstainer . . . . . . . . . . . . . . . . . . . . & $6.7(0.50)$ & $7.3(0.55)$ & $6.9(0.54)$ & $6.1(0.54)$ & $5.0(0.55)$ \\
\hline Former drinker $\ldots \ldots \ldots \ldots \ldots \ldots \ldots \ldots \ldots$ & $13.9(0.94)$ & $17.1(0.99)$ & $12.9(0.89)$ & $9.1(0.80)$ & $6.5(0.70)$ \\
\hline 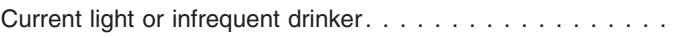 & $9.8(0.45)$ & $9.4(0.41)$ & $7.5(0.40)$ & $7.5(0.39)$ & $5.4(0.31)$ \\
\hline Current moderate or heavier drinker $\ldots \ldots \ldots \ldots \ldots$ & $10.5(0.64)$ & $8.8(0.60)$ & $7.7(0.56)$ & $6.7(0.50)$ & $5.4(0.48)$ \\
\hline \multicolumn{6}{|l|}{ Diabetes status $^{14}$} \\
\hline Yes ....................... & $14.9(1.28)$ & $24.0(1.65)$ & $14.0(1.24)$ & $8.9(1.03)$ & $7.6(1.00)$ \\
\hline 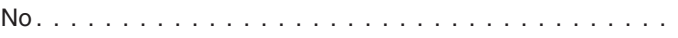 & $9.4(0.32)$ & $8.8(0.29)$ & $7.6(0.27)$ & $7.1(0.27)$ & $5.3(0.24)$ \\
\hline
\end{tabular}

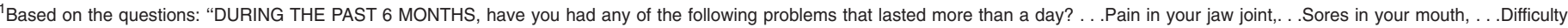
eating or chewing, ... Bad breath, ... .Dry mouth."

2Unknowns for the columns were not included in the denominators when calculating percentages.

${ }^{3}$ Includes other races and Hispanic origin not shown separately and persons with unknown nativity, education, health and dental insurance status, dentition status, current smoking status, current drinking status, and diabetes status.

${ }^{4}$ Persons of Hispanic or Latino origin may be of any race or combination of races. Non-Hispanic or Latino persons of a single race other than groups shown or of multiple race are not shown separately due to small sample sizes.

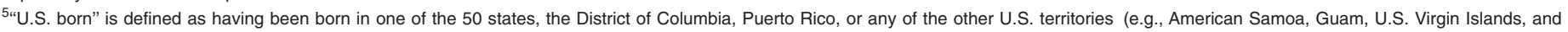

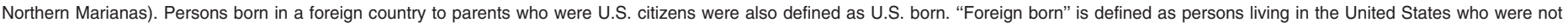
U.S. citizens at birth. All naturalized citizens, legal permanent residents, illegal aliens, and persons on long-term temporary visas (such as students or guest workers) also fall in this category.

${ }^{6}$ Shown only for persons aged 25 and over.

${ }^{7}$ GED is General Educational Development high school equivalency diploma.

${ }^{8}$ Poverty status is based on family income and family size using the U.S. Census Bureau's poverty thresholds for the previous calendar year. "Poor" persons are defined as below the poverty

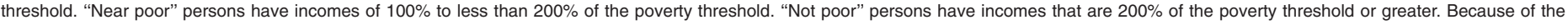
different income questions used in 2007 and beyond, poverty ratio estimates may not be comparable with those from earlier years.

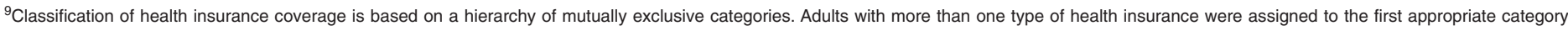

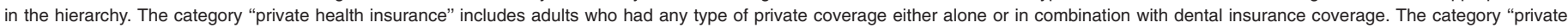

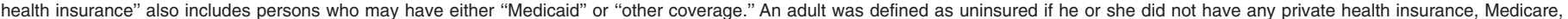

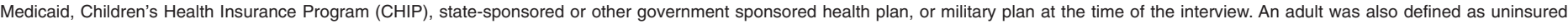

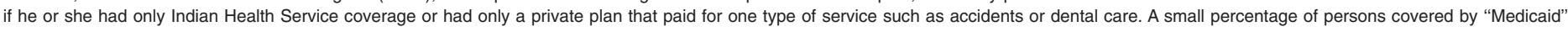

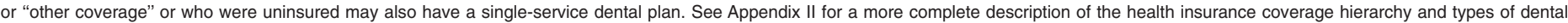
insurance coverage.

${ }^{10}$ Includes adults covered by CHIP or other state-sponsored health plans.

${ }^{11}$ Refers to persons who have lost all of their upper and lower natural teeth.

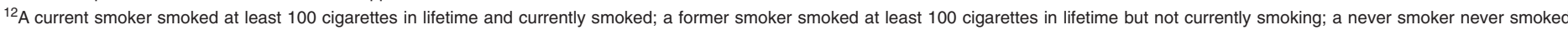
at all or smoked less than 100 cigarettes in lifetime.

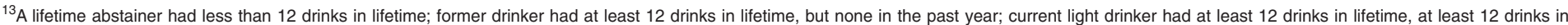

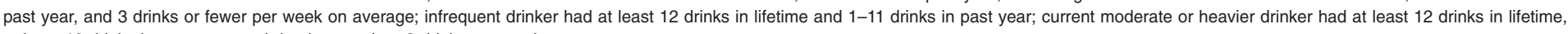
at least 12 drinks in past year, and drank more than 3 drinks per week, on average.

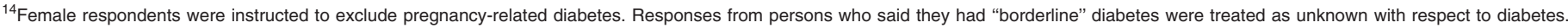
NOTE: Estimates are based on household interviews of a sample of the resident civilian noninstitutionalized population.

SOURCE: CDC/NCHS, National Health Interview Survey, 2008. 
Table 5. Percentages of dentate adults aged 18-64 who had problems with teeth in the past 6 months, by selected demographic and health characteristics: United States, 2008

\begin{tabular}{|c|c|c|c|c|c|c|c|}
\hline \multirow[b]{2}{*}{ Selected characteristic } & \multicolumn{7}{|c|}{ Problem with teeth ${ }^{1}$} \\
\hline & $\begin{array}{l}\text { Toothache } \\
\text { or sensitive } \\
\text { teeth }\end{array}$ & $\begin{array}{c}\text { Stained or } \\
\text { discolored teeth }\end{array}$ & $\begin{array}{l}\text { Broken or } \\
\text { missing teeth }\end{array}$ & $\begin{array}{l}\text { Crooked } \\
\text { teeth }\end{array}$ & $\begin{array}{l}\text { Bleeding } \\
\text { gums }\end{array}$ & $\begin{array}{l}\text { Broken or } \\
\text { missing fillings }\end{array}$ & $\begin{array}{l}\text { Loose teeth } \\
\text { not due } \\
\text { to injury }\end{array}$ \\
\hline & \multicolumn{7}{|c|}{ Percent $^{2}$ (standard error) } \\
\hline Total $^{3}$. & $26.2(0.45)$ & $20.5(0.45)$ & $16.9(0.40)$ & $13.6(0.35)$ & $12.1(0.34)$ & $11.8(0.32)$ & $4.0(0.19)$ \\
\hline \multicolumn{8}{|l|}{ Sex } \\
\hline Male & $23.9(0.65)$ & $20.2(0.61)$ & $17.6(0.60)$ & $13.7(0.50)$ & $11.2(0.47)$ & $11.9(0.47)$ & $4.1(0.28)$ \\
\hline$\ldots \ldots \ldots \ldots \ldots$ & $28.4(0.62)$ & $20.8(0.59)$ & $16.2(0.52)$ & $13.6(0.46)$ & $12.9(0.46)$ & $11.8(0.42)$ & $3.9(0.27)$ \\
\hline \multicolumn{8}{|l|}{ Age in years } \\
\hline $18-34$. & $27.4(0.79)$ & $16.9(0.65)$ & $12.3(0.57)$ & $13.7(0.60)$ & $13.4(0.58)$ & $9.1(0.48)$ & $2.2(0.23)$ \\
\hline $35-44$. & $26.7(0.87)$ & $21.0(0.83)$ & $16.5(0.77)$ & $13.4(0.67)$ & $12.5(0.65)$ & $13.3(0.70)$ & $4.1(0.45)$ \\
\hline $45-54$. & $26.0(0.89)$ & $24.8(0.90)$ & $21.2(0.85)$ & $14.1(0.72)$ & $11.5(0.67)$ & $14.2(0.68)$ & $5.6(0.46)$ \\
\hline $55-64$. & $22.9(0.93)$ & $21.9(0.89)$ & $21.8(0.88)$ & $13.1(0.74)$ & $9.3(0.63)$ & $12.8(0.70)$ & $5.7(0.48)$ \\
\hline \multicolumn{8}{|l|}{ Race/ethnicity ${ }^{4}$} \\
\hline Hispanic or Latino $\ldots \ldots \ldots \ldots$ & $22.0(0.95)$ & $15.9(0.87)$ & $16.0(0.80)$ & $13.3(0.77)$ & $14.1(0.81)$ & $10.6(0.69)$ & $4.5(0.53)$ \\
\hline \multicolumn{8}{|l|}{ Non-Hispanic . . . . . . } \\
\hline White, single race $\ldots \ldots \ldots \ldots \ldots \ldots$ & $27.6(0.59)$ & $23.1(0.57)$ & $17.1(0.51)$ & $14.4(0.45)$ & $11.6(0.42)$ & $12.4(0.42)$ & $3.9(0.25)$ \\
\hline Black, single race $\ldots \ldots \ldots \ldots \ldots \ldots \ldots$ & $26.2(1.11)$ & $15.4(0.92)$ & $19.7(1.05)$ & $11.8(0.83)$ & $12.9(0.92)$ & $12.4(0.77)$ & $4.5(0.47)$ \\
\hline Asian, single race & $20.0(1.52)$ & $11.8(1.23)$ & $9.7(1.16)$ & $8.9(1.09)$ & $9.4(1.00)$ & $6.9(0.79)$ & $2.5(0.49)$ \\
\hline \multicolumn{8}{|l|}{ Nativity ${ }^{5}$} \\
\hline U.S. born. . . . & $27.3(0.51)$ & $22.2(0.50)$ & $17.6(0.45)$ & $14.5(0.40)$ & $12.0(0.38)$ & $12.4(0.36)$ & $4.1(0.21)$ \\
\hline Foreign born. . . . . . & $20.6(0.90)$ & $12.3(0.76)$ & $13.3(0.73)$ & $9.4(0.66)$ & $12.3(0.72)$ & $9.4(0.66)$ & $3.6(0.44)$ \\
\hline \multicolumn{8}{|l|}{ Education $^{6}$} \\
\hline Less than a high school diploma & $29.1(1.29)$ & $27.3(1.36)$ & $28.2(1.32)$ & $17.6(1.13)$ & $14.9(1.01)$ & $15.3(1.01)$ & $8.6(0.75)$ \\
\hline High school diploma or GED ${ }^{7}$. . & $28.3(0.91)$ & $25.8(0.96)$ & $23.7(0.93)$ & $16.0(0.71)$ & $12.5(0.72)$ & $14.5(0.76)$ & $6.5(0.51)$ \\
\hline Some college . . . . . . . . . . & $27.3(0.80)$ & $22.8(0.76)$ & $18.6(0.70)$ & $14.1(0.64)$ & $12.7(0.61)$ & $13.7(0.65)$ & $3.9(0.37)$ \\
\hline Bachelor's degree or higher $\ldots \ldots \ldots \ldots \ldots \ldots$ & $22.2(0.77)$ & $15.2(0.65)$ & $9.8(0.53)$ & $10.3(0.55)$ & $9.7(0.54)$ & $9.8(0.52)$ & $2.0(0.26)$ \\
\hline \multicolumn{8}{|l|}{ Poverty status ${ }^{8}$} \\
\hline Poor. . . . & $32.7(1.29)$ & $25.6(1.35)$ & $25.6(1.31)$ & $17.5(1.03)$ & $16.4(0.99)$ & $16.8(1.06)$ & $7.2(0.63)$ \\
\hline Near poor . . & $31.3(1.15)$ & $25.7(1.12)$ & $24.2(1.08)$ & $16.7(0.90)$ & $15.1(0.84)$ & $15.5(0.86)$ & $6.6(0.60)$ \\
\hline Not poor ... & $24.0(0.50)$ & $18.5(0.48)$ & $13.8(0.43)$ & $12.3(0.40)$ & $10.7(0.37)$ & $10.2(0.35)$ & $2.9(0.20)$ \\
\hline \multicolumn{8}{|l|}{ Health and dental insurance status ${ }^{9}$} \\
\hline Private health insurance $\ldots \ldots$ & $23.2(0.52)$ & $17.6(0.48)$ & $13.0(0.44)$ & $12.2(0.41)$ & $10.2(0.36)$ & $10.0(0.38)$ & $2.7(0.20)$ \\
\hline Dental coverage from single-service plan only . & $27.0(1.06)$ & $20.7(0.97)$ & $13.0(0.77)$ & $14.0(0.76)$ & $11.8(0.75)$ & $10.6(0.67)$ & $2.5(0.32)$ \\
\hline Dental coverage from comprehensive plan only & $21.0(0.94)$ & $15.7(0.77)$ & $13.2(0.83)$ & $10.8(0.74)$ & $9.5(0.62)$ & $10.0(0.76)$ & $2.8(0.38)$ \\
\hline $\begin{array}{l}\text { Dental coverage from both single-service and } \\
\text { comprehensive plans } \ldots \ldots \ldots \ldots \ldots\end{array}$ & $22.6(1.31)$ & $17.5(1.17)$ & $12.0(0.99)$ & $13.1(1.18)$ & $8.9(0.85)$ & $10.6(0.97)$ & $2.6(0.54)$ \\
\hline No dental insurance $\ldots \ldots \ldots \ldots \ldots \ldots$ & $22.7(0.95)$ & $16.8(0.87)$ & $13.6(0.82)$ & $11.3(0.72)$ & $10.0(0.71)$ & $9.5(0.67)$ & $2.9(0.40)$ \\
\hline Medicaid $^{10} \ldots \ldots \ldots$ & $37.2(1.71)$ & $31.3(1.75)$ & $30.4(1.61)$ & $19.0(1.35)$ & $17.3(1.20)$ & $18.5(1.30)$ & $9.1(0.92)$ \\
\hline Other coverage $\ldots \ldots \ldots \ldots \ldots \ldots \ldots \ldots \ldots$ & $34.0(2.23)$ & $29.5(2.19)$ & $26.5(2.09)$ & $18.4(1.75)$ & $14.0(1.76)$ & $17.0(1.71)$ & $8.8(1.24)$ \\
\hline Uninsured $\ldots \ldots \ldots \ldots \ldots \ldots \ldots \ldots \ldots$ & $30.5(0.95)$ & $24.7(1.08)$ & $23.2(0.91)$ & $16.0(0.79)$ & $16.1(0.89)$ & $14.6(0.73)$ & $5.9(0.52)$ \\
\hline \multicolumn{8}{|l|}{ Region } \\
\hline Northeast & $25.8(1.17)$ & $17.8(1.00)$ & $16.1(0.90)$ & $11.7(0.80)$ & $11.0(0.76)$ & $12.5(0.78)$ & $3.6(0.42)$ \\
\hline Midwest . & $28.4(0.99)$ & $21.5(0.93)$ & $16.1(0.82)$ & $13.4(0.80)$ & $10.6(0.69)$ & $11.4(0.78)$ & $3.8(0.43)$ \\
\hline South. . & $25.4(0.73)$ & $22.3(0.80)$ & $18.6(0.72)$ & $14.3(0.56)$ & $12.7(0.61)$ & $11.8(0.49)$ & $4.2(0.29)$ \\
\hline West . & $25.5(0.84)$ & $18.7(0.85)$ & $15.6(0.76)$ & $14.2(0.69)$ & $13.4(0.66)$ & $11.8(0.61)$ & $4.3(0.41)$ \\
\hline \multicolumn{8}{|l|}{ Place of residence } \\
\hline Urban. & $25.8(0.49)$ & $19.0(0.46)$ & $15.8(0.44)$ & $13.1(0.38)$ & $12.2(0.37)$ & $11.5(0.36)$ & $3.9(0.21)$ \\
\hline Rural . & $27.7(1.12)$ & $26.5(1.11)$ & $21.1(0.95)$ & $15.6(0.81)$ & $11.3(0.76)$ & $13.3(0.69)$ & $4.5(0.41)$ \\
\hline \multicolumn{8}{|l|}{ Current smoking status ${ }^{11}$} \\
\hline Current smoker. . & $36.5(1.01)$ & $37.8(1.04)$ & $28.9(0.95)$ & $18.6(0.80)$ & $13.9(0.75)$ & $17.8(0.78)$ & $8.2(0.55)$ \\
\hline Former smoker . . . . . . & $28.2(1.02)$ & $21.3(0.93)$ & $18.7(0.89)$ & $15.0(0.79)$ & $13.2(0.75)$ & $13.7(0.78)$ & $3.9(0.41)$ \\
\hline Never smoker $\ldots \ldots \ldots \ldots \ldots \ldots \ldots \ldots \ldots \ldots \ldots$ & $21.9(0.54)$ & $14.1(0.46)$ & $12.1(0.41)$ & $11.4(0.41)$ & $11.0(0.41)$ & $9.1(0.35)$ & $2.5(0.20)$ \\
\hline
\end{tabular}


Table 5. Percentages of dentate adults aged 18-64 who had problems with teeth in the past 6 months, by selected demographic and health characteristics: United States, 2008-Con.

\begin{tabular}{|c|c|c|c|c|c|c|c|}
\hline \multirow[b]{2}{*}{ Selected characteristic } & \multicolumn{7}{|c|}{ Problem with teeth ${ }^{1}$} \\
\hline & $\begin{array}{c}\text { Toothache } \\
\text { or sensitive } \\
\text { teeth }\end{array}$ & $\begin{array}{c}\text { Stained or } \\
\text { discolored teeth }\end{array}$ & $\begin{array}{l}\text { Broken or } \\
\text { missing teeth }\end{array}$ & $\begin{array}{l}\text { Crooked } \\
\text { teeth }\end{array}$ & $\begin{array}{l}\text { Bleeding } \\
\text { gums }\end{array}$ & $\begin{array}{l}\text { Broken or } \\
\text { missing fillings }\end{array}$ & $\begin{array}{l}\text { Loose teeth } \\
\text { not due } \\
\text { to injury }\end{array}$ \\
\hline Current drinking status ${ }^{12}$ & \multicolumn{7}{|c|}{ Percent $^{2}$ (standard error) } \\
\hline Lifetime abstainer & $20.3(0.92)$ & $13.0(0.77)$ & $13.3(0.77)$ & $10.5(0.69)$ & $10.1(0.68)$ & $8.0(0.56)$ & $2.9(0.36)$ \\
\hline Former drinker $\ldots \ldots \ldots \ldots \ldots \ldots \ldots \ldots \ldots$ & $33.2(1.31)$ & $27.2(1.25)$ & $26.2(1.21)$ & $16.0(0.97)$ & $15.4(1.03)$ & $15.5(1.01)$ & $6.3(0.64)$ \\
\hline Current light or infrequent drinker. . . . . . . . . . & $26.6(0.67)$ & $21.1(0.60)$ & $15.4(0.55)$ & $14.3(0.50)$ & $12.1(0.46)$ & $11.9(0.48)$ & $3.7(0.27)$ \\
\hline Current moderate or heavier drinker $\ldots \ldots \ldots \ldots \ldots$ & $27.6(0.98)$ & $23.1(0.92)$ & $18.6(0.83)$ & $14.2(0.74)$ & $12.3(0.67)$ & $13.4(0.68)$ & $4.5(0.43)$ \\
\hline \multicolumn{8}{|l|}{ Diabetes status ${ }^{13}$} \\
\hline Yes ....................... & $32.3(1.78)$ & $29.4(1.73)$ & $30.3(1.86)$ & $16.2(1.48)$ & $17.5(1.42)$ & $16.5(1.56)$ & $9.8(1.12)$ \\
\hline 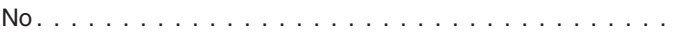 & $25.8(0.47)$ & $20.0(0.46)$ & $16.1(0.41)$ & $13.5(0.36)$ & $11.7(0.35)$ & $11.5(0.33)$ & $3.7(0.19)$ \\
\hline
\end{tabular}

${ }^{1}$ Based on the following questions asked of dentate adults: "DURING THE PAST 6 MONTHS, have you had any of the following problems? ... A toothache or sensitive teeth,. . .Bleeding gums,

..Crooked teeth, ... .Broken or missing teeth, ... Stained or discolored teeth, .. . Loose teeth not due to an injury, ... Broken or missing fillings."

${ }^{2}$ Unknowns for the columns were not included in the denominators when calculating percentages.

${ }^{3}$ Includes other races and Hispanic origin not shown separately and persons with unknown nativity, education, health and dental insurance status, dentition status, current smoking status, current drinking status, and diabetes status.

${ }^{4}$ Persons of Hispanic or Latino origin may be of any race or combination of races. Non-Hispanic or Latino persons of a single race other than groups shown or of multiple race are not shown separately due to small sample sizes.

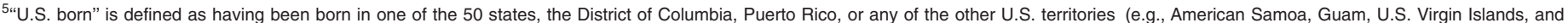

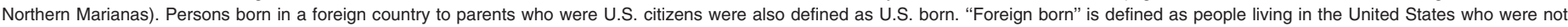
U.S. citizens at birth. All naturalized citizens, legal permanent residents, illegal aliens, and persons on long-term temporary visas (such as students or guest workers) also fall in this category. ${ }^{6}$ Shown only for persons aged 25 and over.

${ }^{7}$ GED is General Educational Development high school equivalency diploma.

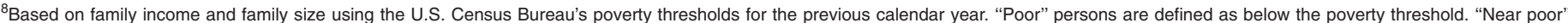
persons have incomes of $100 \%$ to less than $200 \%$ of the poverty threshold. "Not poor" persons have incomes that are $200 \%$ of the poverty threshold or greater. Because of the different income questions used in 2007 and beyond, poverty ratio estimates may not be comparable with those from earlier years.

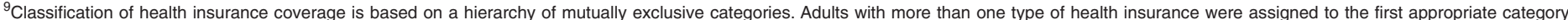

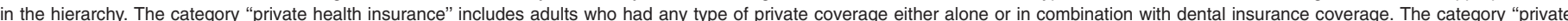
health insurance" also includes persons who may have either "Medicaid" or "other coverage." An adult was defined as uninsured if he or she did not have any private health insurance, Medicare,

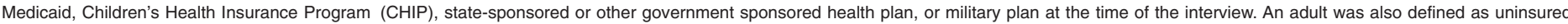

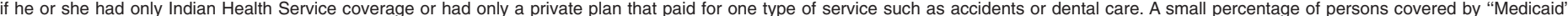

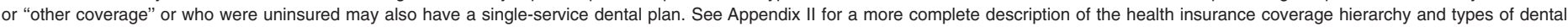
insurance coverage.

${ }^{10}$ Includes adults covered by CHIP or other state-sponsored health plans.

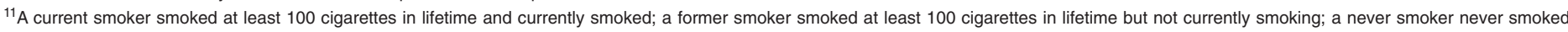
at all or smoked less than 100 cigarettes in lifetime.

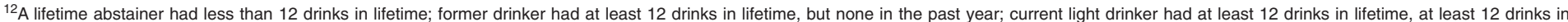

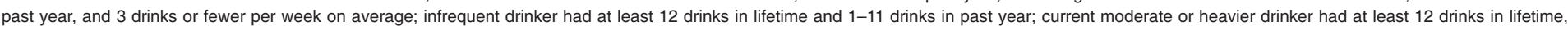
at least 12 drinks in past year, and drank more than 3 drinks per week, on average.

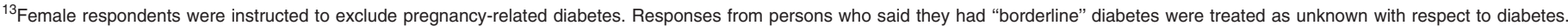
NOTE: Estimates are based on household interviews of a sample of the resident civilian noninstitutionalized population

SOURCE: CDC/NCHS, National Health Interview Survey, 2008. 
Table 6. Percentages of adults aged 18-64 with a mouth or tooth problem who did not see a dentist or other dental health professional in the past 6 months, by reason and by selected demographic and health characteristics: United States, 2008

\begin{tabular}{|c|c|c|c|c|c|c|c|}
\hline \multirow[b]{2}{*}{ Selected characteristic } & \multicolumn{7}{|c|}{ Reason for not seeing a dentist ${ }^{1}$} \\
\hline & $\begin{array}{l}\text { Could not } \\
\text { afford/no } \\
\text { insurance }\end{array}$ & $\begin{array}{l}\text { Didn't think } \\
\text { problem was } \\
\text { important }\end{array}$ & $\begin{array}{l}\text { Problem } \\
\text { went away }\end{array}$ & Fear & $\begin{array}{l}\text { Didn't think } \\
\text { dentist could } \\
\text { fix problem }\end{array}$ & $\begin{array}{l}\text { Waiting for } \\
\text { an appointment }\end{array}$ & $\begin{array}{c}\text { No } \\
\text { transportation }\end{array}$ \\
\hline & \multicolumn{7}{|c|}{ Percent $^{2}$ (standard error) } \\
\hline Total $^{3} \ldots \ldots \ldots \ldots \ldots \ldots \ldots \ldots \ldots \ldots$ & $41.7(0.89)$ & $25.9(0.79)$ & $25.4(0.85)$ & $9.7(0.50)$ & $6.1(0.39)$ & $4.0(0.34)$ & $2.8(0.27)$ \\
\hline \multicolumn{8}{|l|}{ Sex } \\
\hline$\ldots \ldots \ldots \ldots \ldots \ldots \ldots \ldots$ & $39.9(1.28)$ & $29.0(1.20)$ & $26.5(1.13)$ & $9.3(0.74)$ & $5.2(0.51)$ & $3.2(0.45)$ & $2.3(0.37)$ \\
\hline Female . . . . . . . . . . . . . . . . . . & $43.3(1.12)$ & $23.0(0.96)$ & $24.3(1.12)$ & $10.1(0.66)$ & $7.0(0.58)$ & $4.8(0.46)$ & $3.2(0.41)$ \\
\hline \multicolumn{8}{|l|}{ Age in years } \\
\hline 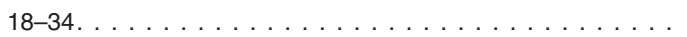 & 43.1 (1.48) & $27.6(1.33)$ & $30.2(1.39)$ & $9.6(0.81)$ & $4.5(0.55)$ & $3.8(0.59)$ & $3.4(0.59)$ \\
\hline 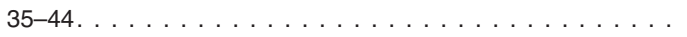 & $41.8(1.67)$ & $25.3(1.54)$ & $22.1(1.45)$ & $10.8(1.04)$ & $6.5(0.82)$ & $4.1(0.65)$ & $2.0(0.44)$ \\
\hline 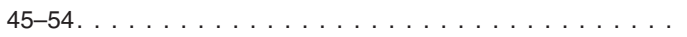 & $44.4(1.81)$ & $25.6(1.50)$ & $22.6(1.47)$ & $9.4(1.05)$ & $7.1(0.86)$ & $4.1(0.60)$ & $2.2(0.41)$ \\
\hline 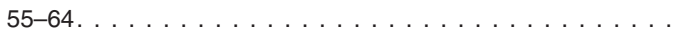 & $34.0(1.86)$ & $23.2(1.66)$ & $22.3(1.65)$ & $8.9(1.07)$ & $8.2(0.95)$ & $4.2(0.73)$ & $3.1(0.64)$ \\
\hline \multicolumn{8}{|l|}{ Race/ethnicity ${ }^{4}$} \\
\hline Hispanic or Latino . . . . . . . . . . . . . . . . . . . . . . . & $55.7(2.10)$ & $21.4(1.57)$ & $22.0(1.66)$ & $11.7(1.13)$ & $5.7(0.85)$ & $3.6(0.61)$ & $3.6(0.64)$ \\
\hline \multicolumn{8}{|l|}{ Non-Hispanic . . . . . . . . . . . . . . . . . . . . . . . } \\
\hline 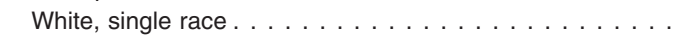 & $38.5(1.07)$ & $28.4(1.05)$ & $25.9(1.10)$ & $9.1(0.63)$ & $6.6(0.50)$ & $3.9(0.43)$ & $2.0(0.31)$ \\
\hline Black, single race $\ldots \ldots \ldots \ldots \ldots \ldots$ & $44.5(2.07)$ & $18.7(1.68)$ & $22.7(1.88)$ & $12.3(1.33)$ & $4.9(0.90)$ & $5.2(0.86)$ & $5.2(0.91)$ \\
\hline Asian, single race $\ldots \ldots \ldots \ldots \ldots \ldots$ & $28.1(3.30)$ & $28.9(3.41)$ & $37.5(3.58)$ & $6.8(1.66)$ & *4.7 (1.45) & $4.2(1.17)$ & $\dagger$ \\
\hline \multicolumn{8}{|l|}{ Nativity $^{5}$} \\
\hline U.S. born. . . . . . . . . . . . . . . . . . . & $39.8(0.94)$ & $26.6(0.86)$ & $25.7(0.95)$ & $9.7(0.56)$ & $6.3(0.43)$ & $4.0(0.38)$ & $2.7(0.30)$ \\
\hline Foreign born. . . . . . . . . . . . . . . . . . & $52.6(2.15)$ & $21.6(1.62)$ & $23.5(1.73)$ & $9.6(1.11)$ & $5.4(0.92)$ & $4.3(0.77)$ & $3.0(0.57)$ \\
\hline \multicolumn{8}{|l|}{ Education $^{6}$} \\
\hline Less than a high school diploma . . . . . . . . . . . . . . & $58.4(2.10)$ & $18.1(1.72)$ & $20.2(1.86)$ & $13.0(1.31)$ & $3.6(0.69)$ & $3.9(0.71)$ & $5.6(0.89)$ \\
\hline High school diploma or $\mathrm{GED}^{7} \ldots \ldots \ldots \ldots \ldots \ldots$ & $47.7(1.65)$ & $23.6(1.47)$ & $20.2(1.35)$ & $13.1(1.11)$ & $6.3(0.81)$ & $2.6(0.43)$ & $2.4(0.46)$ \\
\hline Some college . . . . . . . . . . . . . . . . . . & $42.8(1.56)$ & $24.2(1.34)$ & $23.7(1.41)$ & $7.1(0.75)$ & $6.8(0.74)$ & $4.6(0.64)$ & $1.7(0.34)$ \\
\hline Bachelor's degree or higher . . . . . . . . . . . . . & $23.4(1.47)$ & $33.1(1.74)$ & $30.7(1.73)$ & $7.3(0.93)$ & $8.0(0.96)$ & $4.6(0.76)$ & $\dagger$ \\
\hline \multicolumn{8}{|l|}{ Poverty status ${ }^{8}$} \\
\hline 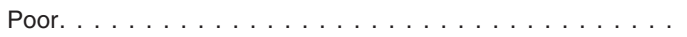 & $59.7(2.20)$ & $17.8(1.61)$ & $19.3(1.45)$ & $11.1(1.11)$ & $5.7(0.91)$ & $4.3(0.66)$ & $8.5(1.10)$ \\
\hline Near poor . . . . . . . . . . . . . . . . . & $59.6(1.72)$ & $19.1(1.43)$ & $20.8(1.42)$ & $10.1(1.04)$ & $5.5(0.81)$ & $3.4(0.66)$ & $4.0(0.85)$ \\
\hline 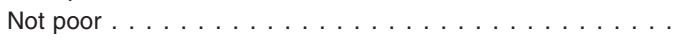 & $31.0(1.02)$ & $30.3(1.09)$ & $28.4(1.14)$ & $9.2(0.63)$ & $6.5(0.50)$ & $4.2(0.47)$ & $0.8(0.20)$ \\
\hline \multicolumn{8}{|l|}{ Health and dental insurance status ${ }^{9}$} \\
\hline Private health insurance $\ldots \ldots \ldots \ldots \ldots \ldots$ & $24.1(0.96)$ & $31.9(1.12)$ & $29.0(1.15)$ & $9.7(0.68)$ & $7.4(0.56)$ & $4.7(0.51)$ & $0.7(0.18)$ \\
\hline Dental coverage from single-service plan only $\ldots \ldots \ldots$ & $17.6(1.64)$ & $37.7(2.18)$ & $31.7(2.17)$ & $9.8(1.30)$ & $9.3(1.22)$ & $3.8(0.76)$ & $\dagger$ \\
\hline Dental coverage from comprehensive plan only $\ldots \ldots \ldots$ & $18.2(1.56)$ & $30.2(1.88)$ & $28.3(1.91)$ & $9.9(1.31)$ & $7.0(0.97)$ & $6.8(1.23)$ & ${ }^{\star} 0.5(0.21)$ \\
\hline $\begin{array}{l}\text { Dental coverage from both single-service and } \\
\text { comprehensive plans } \ldots \ldots \ldots \ldots \ldots \ldots \ldots \ldots \ldots \ldots\end{array}$ & $18.4(2.32)$ & $30.6(2.82)$ & $28.3(2.80)$ & $9.9(1.82)$ & $6.4(1.52)$ & $4.9(1.34)$ & $\dagger$ \\
\hline No dental insurance. . . . . . . . . . . . . . . . . & $40.1(2.20)$ & $27.8(2.09)$ & $27.3(1.97)$ & $9.0(1.22)$ & $6.8(0.95)$ & $3.1(0.68)$ & ${ }^{*} 0.9(0.44)$ \\
\hline Medicaid $^{10} \ldots \ldots \ldots \ldots \ldots \ldots \ldots \ldots \ldots$ & $47.6(2.42)$ & $17.7(1.91)$ & $20.9(2.10)$ & $16.4(1.82)$ & $8.7(1.49)$ & $6.9(1.17)$ & $10.3(1.54)$ \\
\hline Other coverage $\ldots \ldots \ldots \ldots \ldots \ldots \ldots \ldots$ & $49.8(3.49)$ & $19.3(2.81)$ & $26.9(3.43)$ & $7.0(1.69)$ & $7.1(1.74)$ & *3.0 (1.26) & *3.1 (0.96) \\
\hline 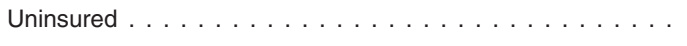 & $75.5(1.37)$ & $16.7(1.24)$ & $18.9(1.34)$ & $7.7(0.74)$ & $2.4(0.41)$ & $1.8(0.39)$ & $4.1(0.69)$ \\
\hline \multicolumn{8}{|l|}{ Region } \\
\hline 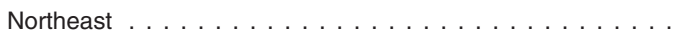 & $33.6(2.06)$ & $27.2(2.46)$ & $24.8(2.19)$ & $10.1(1.29)$ & $5.9(1.04)$ & $3.8(0.86)$ & $3.4(0.71)$ \\
\hline 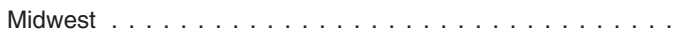 & $35.6(1.76)$ & $31.1(1.81)$ & $32.5(1.84)$ & $8.0(0.94)$ & $7.5(0.86)$ & $4.1(0.72)$ & $2.0(0.50)$ \\
\hline South . . . . . . . . . . . . . . . . . . & $48.1(1.55)$ & $21.0(1.13)$ & $21.1(1.28)$ & $10.1(0.77)$ & $4.7(0.58)$ & $3.6(0.42)$ & $3.3(0.52)$ \\
\hline 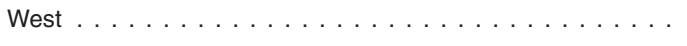 & $43.0(1.72)$ & $27.4(1.41)$ & $24.8(1.72)$ & $10.7(1.17)$ & $7.1(0.85)$ & $4.8(0.90)$ & $2.4(0.44)$ \\
\hline \multicolumn{8}{|l|}{ Place of residence } \\
\hline 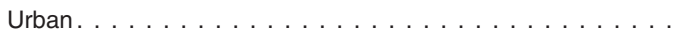 & $41.5(1.02)$ & $25.7(0.87)$ & $26.6(0.95)$ & $10.3(0.58)$ & $6.2(0.44)$ & $4.2(0.39)$ & $2.9(0.34)$ \\
\hline 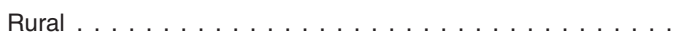 & $42.1(1.76)$ & $26.7(1.92)$ & $20.9(1.68)$ & $7.8(0.95)$ & $5.9(0.86)$ & $3.5(0.61)$ & $2.3(0.53)$ \\
\hline \multicolumn{8}{|l|}{ Dentition status } \\
\hline Edentulous $^{11} \ldots \ldots \ldots \ldots$ & $39.8(4.48)$ & $17.9(4.50)$ & $28.2(4.93)$ & *4.9(1.52) & $11.8(2.32)$ & *2.6 (1.24) & *3.3 (1.23) \\
\hline 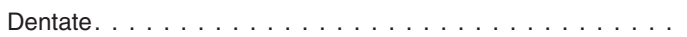 & $41.7(0.91)$ & $26.2(0.79)$ & $25.2(0.85)$ & $9.9(0.51)$ & $5.9(0.39)$ & $4.1(0.35)$ & $2.7(0.28)$ \\
\hline
\end{tabular}

See footnotes at end of table. 
Table 6. Percentages of adults aged 18-64 with a mouth or tooth problem who did not see a dentist or other dental health professional in the past 6 months, by reason and by selected demographic and health characteristics: United States, 2008-Con.

\begin{tabular}{|c|c|c|c|c|c|c|c|}
\hline \multirow[b]{2}{*}{ Selected characteristic } & \multicolumn{7}{|c|}{ Reason for not seeing a dentist ${ }^{1}$} \\
\hline & $\begin{array}{l}\text { Could not } \\
\text { afford/no } \\
\text { insurance }\end{array}$ & $\begin{array}{l}\text { Didn't think } \\
\text { problem was } \\
\text { important }\end{array}$ & $\begin{array}{c}\text { Problem } \\
\text { went away }\end{array}$ & Fear & $\begin{array}{l}\text { Didn't think } \\
\text { dentist could } \\
\text { fix problem }\end{array}$ & $\begin{array}{c}\text { Waiting for } \\
\text { an appointment }\end{array}$ & $\begin{array}{c}\text { No } \\
\text { transportation }\end{array}$ \\
\hline Current smoking status ${ }^{12}$ & \multicolumn{7}{|c|}{ Percent $^{2}$ (standard error) } \\
\hline 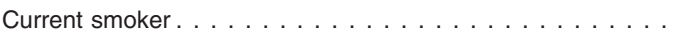 & $55.5(1.60)$ & $22.1(1.35)$ & $19.8(1.28)$ & $10.5(0.84)$ & $5.6(0.65)$ & $3.6(0.63)$ & $4.3(0.64)$ \\
\hline 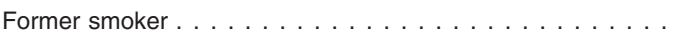 & $36.4(1.79)$ & $27.0(1.64)$ & $23.0(1.59)$ & $9.0(1.13)$ & $8.1(1.01)$ & $4.7(0.79)$ & $1.7(0.43)$ \\
\hline Never smoker . . . . . . . . . . . . . . . . . & $34.9(1.12)$ & $27.9(1.09)$ & $29.7(1.20)$ & $9.5(0.69)$ & $5.7(0.53)$ & $4.0(0.43)$ & $2.2(0.35)$ \\
\hline \multicolumn{8}{|l|}{ Current drinking status ${ }^{13}$} \\
\hline Lifetime abstainer . . . . . . . . . . . . . . . . . . . & $43.8(1.97)$ & $22.2(1.93)$ & $25.1(2.03)$ & $8.1(1.09)$ & $5.5(0.95)$ & $6.3(1.15)$ & $4.0(0.80)$ \\
\hline 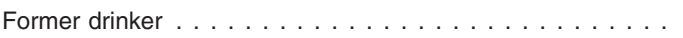 & $49.1(2.28)$ & $20.0(1.73)$ & $18.0(1.71)$ & $12.9(1.33)$ & $7.2(1.05)$ & $3.3(0.66)$ & $3.9(0.73)$ \\
\hline Current light or infrequent drinker. . . . . . . . . . & $39.5(1.26)$ & $26.8(1.14)$ & $25.7(1.15)$ & $9.9(0.75)$ & $7.0(0.63)$ & $3.7(0.45)$ & $1.7(0.30)$ \\
\hline Current moderate or heavier drinker . . . . . . . . . . . & $39.8(1.66)$ & $30.6(1.66)$ & $29.1(1.63)$ & $8.5(0.97)$ & $4.4(0.61)$ & $3.6(0.62)$ & $3.0(0.66)$ \\
\hline \multicolumn{8}{|l|}{ Diabetes status ${ }^{14}$} \\
\hline Yes ...................... & $48.3(2.96)$ & $18.7(2.31)$ & $19.2(2.27)$ & $11.2(1.55)$ & $8.5(1.47)$ & $3.4(0.91)$ & $5.2(1.14)$ \\
\hline 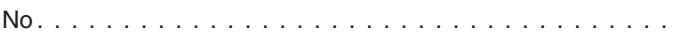 & $41.1(0.91)$ & $26.5(0.82)$ & $25.9(0.89)$ & $9.6(0.53)$ & $5.9(0.41)$ & $4.1 \quad(0.36)$ & $2.6(0.28)$ \\
\hline
\end{tabular}

* Estimate has a relative standard error of greater than $30 \%$ and less than or equal to $50 \%$ and should be used with caution as it does not meet standards of reliability or precision.

† Estimate has a relative standard error greater than $50 \%$ and is not shown.

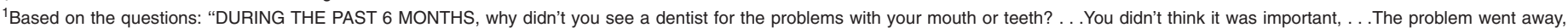

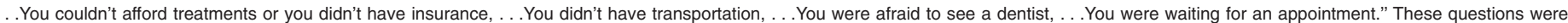
asked only of adults who indicated that they had a mouth or tooth problem as shown in Tables 4 and 5.

${ }^{2}$ Unknowns for the columns were not included in the denominators when calculating percentages.

${ }^{3}$ Includes other races and Hispanic origin not shown separately and persons with unknown nativity, education, health and dental insurance status, dentition status, current smoking status, current drinking status, and diabetes status.

${ }^{4}$ Persons of Hispanic or Latino origin may be of any race or combination of races. Non-Hispanic or Latino persons of a single race other than groups shown or of multiple race are not shown separately due to small sample sizes.

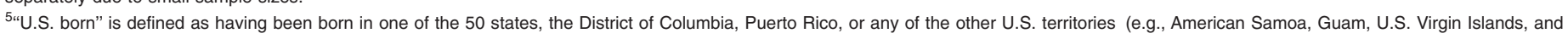

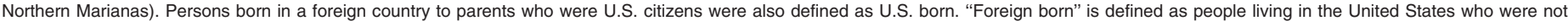
U.S. citizens at birth. All naturalized citizens, legal permanent residents, illegal aliens, and persons on long-term temporary visas (such as students or guest workers) also fall in this category.

${ }^{6}$ Shown only for persons aged 25 and over.

${ }^{7}$ GED is General Educational Development high school equivalency diploma.

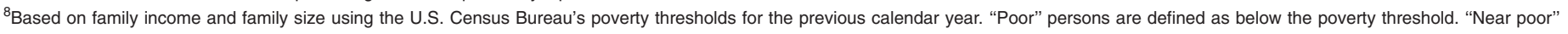
persons have incomes of $100 \%$ to less than $200 \%$ of the poverty threshold. "Not poor" persons have incomes that are $200 \%$ of the poverty threshold or greater. Because of the different income questions used in 2007 and beyond, poverty ratio estimates may not be comparable with those from earlier years.

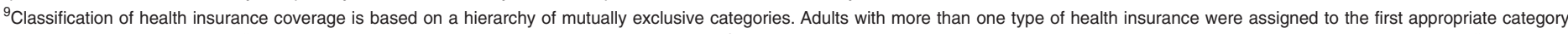

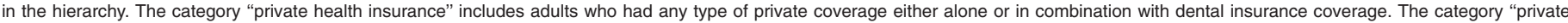

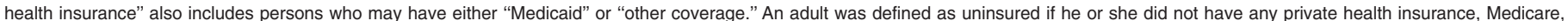

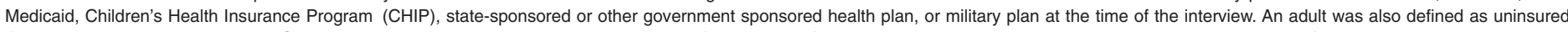

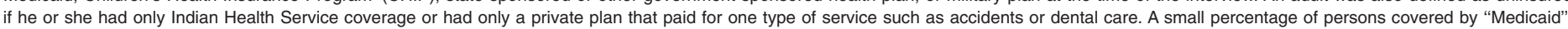

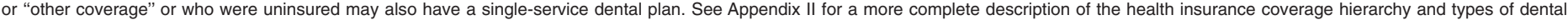
insurance coverage.

${ }^{10}$ Includes adults covered by CHIP or other state-sponsored health plans.

${ }^{11}$ Refers to persons who have lost all of their upper and lower natural teeth.

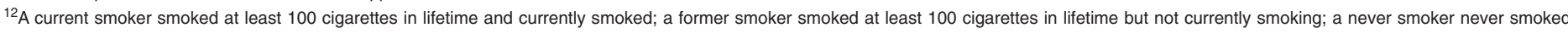
at all or smoked less than 100 cigarettes in lifetime.

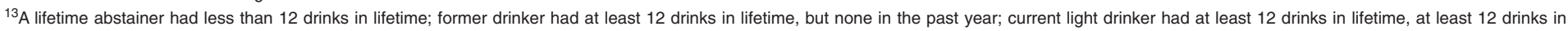

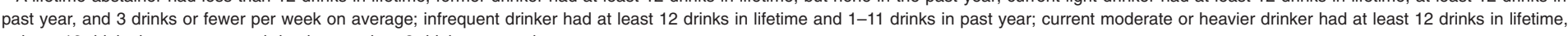
at least 12 drinks in past year, and drank more than 3 drinks per week, on average.

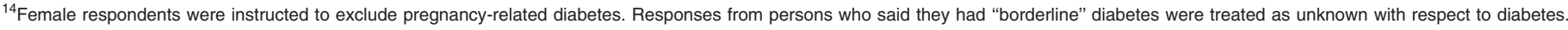
NOTE: Estimates are based on household interviews of a sample of the resident civilian noninstitutionalized population.

SOURCE: CDC/NCHS, National Health Interview Survey, 2008. 
Table 7. Percentages of dentate adults aged 18-64 who had an unmet dental need due to cost in the past 12 months, dentate adults with no mouth or teeth problems, with one or more mouth or teeth problems, and with toothache as a problem, by selected demographic and health characteristics: United States, 2008

\begin{tabular}{|c|c|c|c|c|}
\hline \multirow[b]{2}{*}{ Selected characteristic } & \multicolumn{4}{|c|}{ Unmet dental need due to cost ${ }^{1}$} \\
\hline & $\begin{array}{l}\text { All dentate } \\
\text { adults }\end{array}$ & $\begin{array}{l}\text { Dentate adults } \\
\text { with no } \\
\text { mouth or } \\
\text { teeth problems }\end{array}$ & $\begin{array}{l}\text { Dentate adults } \\
\text { with one or } \\
\text { more mouth } \\
\text { or teeth problems }\end{array}$ & $\begin{array}{l}\text { Dentate adults } \\
\text { with toothache } \\
\text { as a problem }{ }^{2}\end{array}$ \\
\hline & \multicolumn{4}{|c|}{ Percent $^{3}$ (standard error) } \\
\hline$\ldots \ldots \ldots \ldots \ldots$ & $16.1(0.39)$ & $6.8(0.37)$ & $25.3(0.64)$ & $32.7(0.96)$ \\
\hline \multicolumn{5}{|l|}{ Sex } \\
\hline$\ldots \ldots \ldots \ldots \ldots \ldots \ldots \ldots \ldots$ & $14.0(0.52)$ & $6.0(0.50)$ & $22.6(0.85)$ & $30.1(1.35)$ \\
\hline$\ldots \ldots \ldots \ldots \ldots \ldots \ldots \ldots \ldots$ & $18.0(0.52)$ & $7.6(0.49)$ & $27.8(0.86)$ & $34.9(1.30)$ \\
\hline \multicolumn{5}{|l|}{ Age in years } \\
\hline 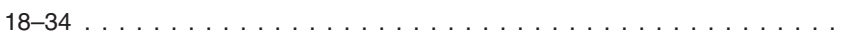 & $17.4(0.65)$ & $7.3(0.60)$ & $28.4(1.07)$ & $35.7(1.49)$ \\
\hline 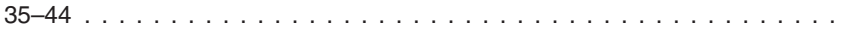 & $15.4(0.71)$ & $7.2(0.74)$ & $23.5(1.16)$ & $29.6(1.76)$ \\
\hline 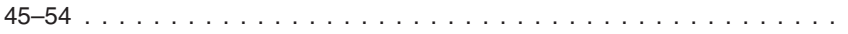 & $16.7(0.76)$ & $5.9(0.66)$ & $26.5(1.24)$ & $35.1(2.00)$ \\
\hline 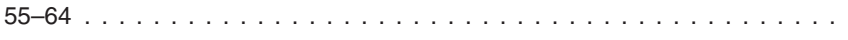 & $13.1(0.76)$ & $6.4(0.80)$ & $19.5(1.21)$ & $25.9(1.93)$ \\
\hline \multicolumn{5}{|l|}{ Race/ethnicity ${ }^{5}$} \\
\hline$\ldots \ldots \ldots \ldots \ldots \ldots \ldots \ldots \ldots$ & $20.9(1.10)$ & $12.0(1.25)$ & $31.1(1.60)$ & $39.3(2.45)$ \\
\hline \multicolumn{5}{|l|}{ Non-Hispanic. . . . . . . . . . } \\
\hline$\ldots \ldots \ldots \ldots \ldots \ldots \ldots \ldots \ldots$ & $15.0(0.48)$ & $5.4(0.40)$ & $24.0(0.78)$ & $31.6(1.20)$ \\
\hline Black, single race. $\ldots \ldots \ldots \ldots \ldots \ldots \ldots \ldots \ldots \ldots$ & $18.2(0.94)$ & $8.8(0.97)$ & $28.4(1.60)$ & $34.1(2.33)$ \\
\hline Asian, single race $\ldots \ldots \ldots \ldots \ldots \ldots \ldots \ldots \ldots$ & $8.9(1.04)$ & $4.3(0.98)$ & $15.6(2.04)$ & $20.9(3.33)$ \\
\hline \multicolumn{5}{|l|}{ Nativity ${ }^{6}$} \\
\hline$\ldots \ldots \ldots \ldots \ldots$ & $16.0(0.43)$ & $6.1(0.37)$ & $25.2(0.71)$ & $33.0(1.05)$ \\
\hline$\ldots \ldots \ldots \ldots \ldots \ldots \ldots \ldots$ & $16.5(0.96)$ & $9.5(1.02)$ & $26.0(1.50)$ & $30.8(2.23)$ \\
\hline \multicolumn{5}{|l|}{ Education $^{7}$} \\
\hline Less than a high school diploma $\ldots \ldots \ldots \ldots \ldots \ldots \ldots$ & $24.9(1.27)$ & $11.7(1.48)$ & $35.7(1.78)$ & $44.1(2.69)$ \\
\hline High school diploma or $\mathrm{GED}^{8} \ldots \ldots \ldots \ldots \ldots \ldots \ldots \ldots \ldots \ldots \ldots$ & $20.0(0.82)$ & $8.1(0.75)$ & $30.3(1.30)$ & $38.9(1.95)$ \\
\hline 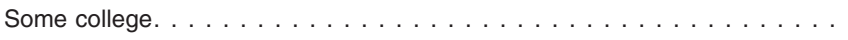 & $18.2(0.76)$ & $7.8(0.72)$ & $28.1(1.21)$ & $35.6(1.74)$ \\
\hline Bachelor's degree or higher $\ldots \ldots \ldots \ldots \ldots \ldots \ldots$ & $9.3(0.53)$ & $4.4(0.53)$ & $15.1(0.94)$ & $20.8(1.59)$ \\
\hline \multicolumn{5}{|l|}{ Poverty status ${ }^{9}$} \\
\hline$\ldots \ldots \ldots \ldots \ldots \ldots$ & $27.9(1.38)$ & $11.8(1.36)$ & $40.6(1.89)$ & $50.5(2.65)$ \\
\hline$\ldots \ldots \ldots \ldots \ldots$ & $28.4(1.21)$ & $13.2(1.33)$ & $40.3(1.61)$ & $48.1(2.25)$ \\
\hline$\ldots \ldots \ldots \ldots$ & $11.3(0.39)$ & $4.9(0.37)$ & $18.4(0.69)$ & $24.2(1.10)$ \\
\hline \multicolumn{5}{|l|}{ Health and dental insurance status ${ }^{10}$} \\
\hline Private health insurance $\ldots \ldots \ldots \ldots \ldots \ldots \ldots \ldots$ & $8.9(0.33)$ & $3.5(0.29)$ & $14.9(0.62)$ & $19.5(1.00)$ \\
\hline Dental coverage from single-service plan only $\ldots \ldots \ldots \ldots \ldots \ldots$ & $7.9(0.59)$ & $2.5(0.47)$ & $12.9(1.02)$ & $17.1(1.65)$ \\
\hline Dental coverage from comprehensive plan only $\ldots \ldots \ldots \ldots \ldots \ldots$ & $6.9(0.55)$ & $2.6(0.38)$ & $12.5(1.15)$ & $16.4(1.91)$ \\
\hline Dental coverage from both single-service and comprehensive plans $\ldots$. & $7.0(0.75)$ & $2.7(0.68)$ & $11.9(1.39)$ & $17.1(2.33)$ \\
\hline No dental insurance $\ldots \ldots \ldots \ldots \ldots \ldots \ldots \ldots \ldots \ldots$ & $13.9(0.84)$ & $6.3(0.78)$ & $22.7(1.44)$ & $28.4(2.20)$ \\
\hline$\ldots \ldots \ldots \ldots$ & $23.2(1.41)$ & $9.5(1.46)$ & $32.5(2.07)$ & $42.0(2.81)$ \\
\hline Other coverage $\ldots \ldots \ldots \ldots \ldots \ldots \ldots \ldots \ldots \ldots \ldots \ldots$ & $23.6(2.06)$ & $8.2(2.03)$ & $33.7(2.98)$ & $42.2(4.27)$ \\
\hline 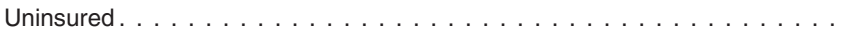 & $36.8(1.15)$ & $18.9(1.36)$ & $51.8(1.44)$ & $61.7(1.86)$ \\
\hline \multicolumn{5}{|l|}{ Region } \\
\hline$\ldots \ldots \ldots \ldots \ldots$ & $12.1(0.73)$ & $5.0(0.65)$ & $19.7(1.27)$ & $25.3(2.02)$ \\
\hline . & $15.1(0.86)$ & $6.5(0.72)$ & $22.8(1.39)$ & $30.3(2.10)$ \\
\hline 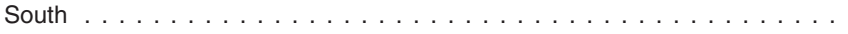 & $18.0(0.72)$ & $7.6(0.66)$ & $28.5(1.09)$ & $36.3(1.57)$ \\
\hline 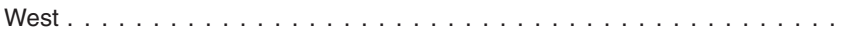 & $17.0(0.76)$ & $7.2(0.80)$ & $27.1(1.30)$ & $35.4(1.99)$ \\
\hline \multicolumn{5}{|l|}{ Place of residence } \\
\hline Urban ............ & $16.0(0.44)$ & $7.1(0.42)$ & $25.1(0.72)$ & $31.9(1.08)$ \\
\hline$\ldots \ldots \ldots \ldots \ldots \ldots \ldots \ldots \ldots$ & $16.4(0.81)$ & $5.6(0.67)$ & $26.0(1.26)$ & $35.8(2.11)$ \\
\hline \multicolumn{5}{|l|}{ Current smoking status ${ }^{12}$} \\
\hline Current smoker $\ldots \ldots \ldots \ldots \ldots \ldots \ldots \ldots \ldots \ldots \ldots \ldots \ldots$ & $26.0(0.96)$ & $9.0(0.89)$ & $35.7(1.29)$ & $45.6(1.78)$ \\
\hline Former smoker. $\ldots \ldots \ldots \ldots \ldots \ldots \ldots$ & $16.0(0.83)$ & $7.1(0.85)$ & $23.6(1.28)$ & $30.2(1.96)$ \\
\hline Never smoker $\ldots \ldots \ldots \ldots \ldots \ldots \ldots \ldots \ldots \ldots \ldots \ldots \ldots \ldots \ldots$ & $12.5(0.43)$ & $6.2(0.43)$ & $20.4(0.79)$ & $25.9(1.25)$ \\
\hline
\end{tabular}


Table 7. Percentages of dentate adults aged 18-64 who had an unmet dental need due to cost in the past 12 months, dentate adults with no mouth or teeth problems, with one or more mouth or teeth problems, and with toothache as a problem, by selected demographic and health characteristics: United States, 2008-Con.

\begin{tabular}{|c|c|c|c|c|}
\hline \multirow[b]{2}{*}{ Selected characteristic } & \multicolumn{4}{|c|}{ Unmet dental need due to cost ${ }^{1}$} \\
\hline & $\begin{array}{l}\text { All dentate } \\
\text { adults }\end{array}$ & $\begin{array}{l}\text { Dentate adults } \\
\text { with no } \\
\text { mouth or } \\
\text { teeth problems }{ }^{2}\end{array}$ & $\begin{array}{c}\text { Dentate adults } \\
\text { with one or } \\
\text { more mouth } \\
\text { or teeth problems } 2\end{array}$ & $\begin{array}{l}\text { Dentate adults } \\
\text { with toothache } \\
\text { as a problem }{ }^{2}\end{array}$ \\
\hline Current drinking status ${ }^{13}$ & \multicolumn{4}{|c|}{ Percent $^{3}$ (standard error) } \\
\hline Lifetime abstainer $\ldots \ldots \ldots \ldots \ldots \ldots$ & $12.5(0.74)$ & $6.7(0.77)$ & $21.2(1.36)$ & $23.3(1.94)$ \\
\hline 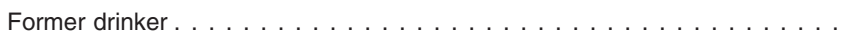 & $22.3(1.16)$ & $7.7(1.08)$ & $33.2(1.78)$ & $42.8(2.52)$ \\
\hline Current light or infrequent drinker $\ldots \ldots \ldots \ldots \ldots \ldots \ldots \ldots \ldots \ldots \ldots$ & $15.9(0.52)$ & $6.5(0.49)$ & $24.8(0.86)$ & $31.8(1.32)$ \\
\hline Current moderate or heavier drinker. $\ldots \ldots \ldots \ldots \ldots \ldots$ & $16.5(0.81)$ & $7.3(0.81)$ & $24.5(1.26)$ & $34.5(1.98)$ \\
\hline \multicolumn{5}{|l|}{ Diabetes status ${ }^{14}$} \\
\hline 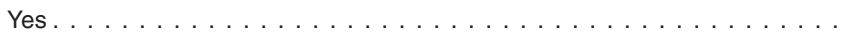 & $20.8(1.49)$ & $7.8(1.57)$ & $28.3(2.03)$ & $36.4(3.28)$ \\
\hline 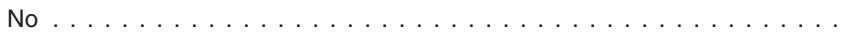 & $15.8(0.40)$ & $6.8(0.37)$ & $25.1(0.65)$ & $32.5(0.98)$ \\
\hline
\end{tabular}

${ }^{1}$ Based on the question: "DURING THE PAST 12 MONTHS, was there any time when you needed any of the following, but didn't get it because you couldn't afford it? ... Dental care (including check-ups)." This table includes dentate adults only.

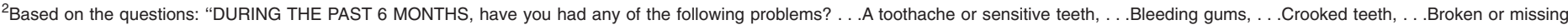
teeth, ... .Stained or discolored teeth, . . .Loose teeth not due to an injury, . . .Broken or missing fillings."

${ }^{3}$ Unknowns for the columns were not included in the denominators when calculating percentages.

${ }^{4}$ Includes other races and Hispanic origin not shown separately and persons with unknown nativity, education, health and dental insurance status, dentition status, current smoking status, current drinking status, and diabetes status.

${ }^{5}$ Persons of Hispanic or Latino origin may be of any race or combination of races. Non-Hispanic or Latino persons of a single race other than groups shown or of multiple race are not shown separately due to small sample sizes.

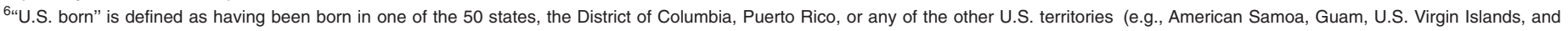

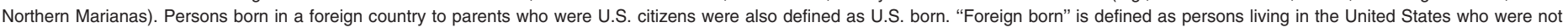
U.S. citizens at birth. All naturalized citizens, legal permanent residents, illegal aliens, and persons on long-term temporary visas (such as students or guest workers) also fall in this category.

${ }^{7}$ Shown only for persons aged 25 and over.

${ }^{8}$ GED is General Educational Development high school equivalency diploma.

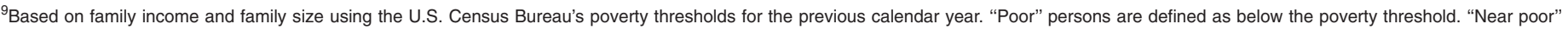
persons have incomes of $100 \%$ to less than $200 \%$ of the poverty threshold. "Not poor" persons have incomes that are $200 \%$ of the poverty threshold or greater. Because of the different income questions used in 2007 and beyond, poverty ratio estimates may not be comparable with those from earlier years.

${ }^{10}$ Classification of health insurance coverage is based on a hierarchy of mutually exclusive categories. Adults with more than one type of health insurance were assigned to the first appropriate

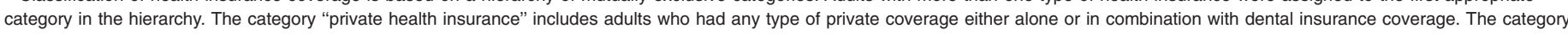
"private health insurance" also includes persons who may have either "Medicaid" or "other coverage." An adult was defined as uninsured if he or she did not have any private health insurance,

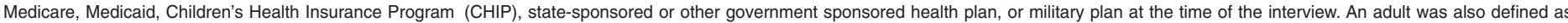

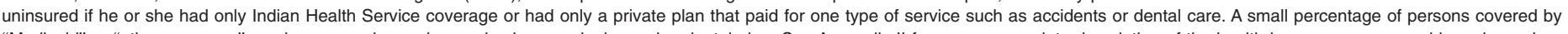

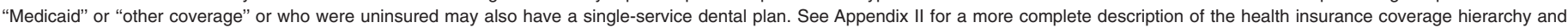
types of dental insurance coverage.

${ }^{11}$ Includes adults covered by CHIP or other state-sponsored health plans.

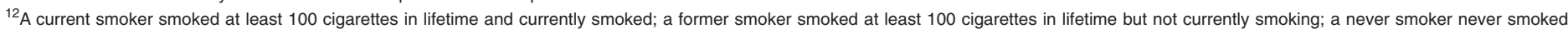
at all or smoked less than 100 cigarettes in lifetime.

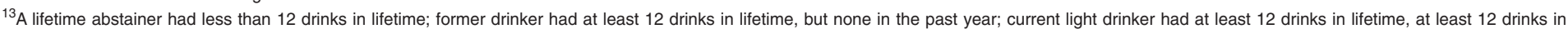

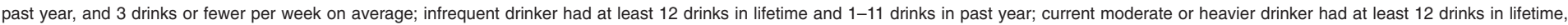
at least 12 drinks in past year, and drank more than 3 drinks per week, on average.

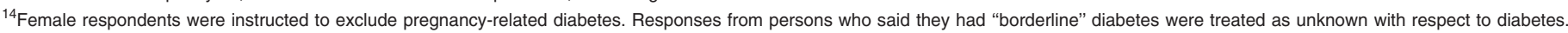
NOTE: Estimates are based on household interviews of a sample of the resident civilian noninstitutionalized population.

SOURCE: CDC/NCHS, National Health Interview Survey, 2008. 


\section{Appendix I. Technical Notes on Methods}

\section{Sample Design}

The National Health Interview Survey (NHIS) is a cross-sectional household interview survey of the U.S. civilian noninstitutionalized population. Data are collected continuously throughout the year in all 50 states and the District of Columbia. NHIS uses a multistage, clustered sample design to collect information for a variety of health indicators. Information on basic health topics is collected for all household members, if necessary, by proxy from one adult family member. Additional information is collected for one randomly selected child (the "sample child") and one randomly selected adult (the "sample adult") in each family. Self response is required for the Sample Adult questionnaire except in rare cases where sample adults are physically or mentally incapable of responding for themselves. Interviews are conducted in the home using computer-assisted personal interviewing (CAPI), with follow-up telephone interviewing permitted, if necessary.

The 2008 NHIS sample design oversamples households with black, Hispanic, and Asian persons. Black, Hispanic, and Asian persons aged 65 and over also have an increased chance of being selected as a sample adult.

\section{Response Rates}

In 2008, NHIS interviews were completed in 28,790 households and 29,421 families, with 21,781 adults aged 18-64 completing the Sample Adult questionnaire. The conditional response rate for the 2008 Sample Adult section was $74.2 \%$, and the final response rate (which takes into account household and family nonresponse) was $62.6 \%$. The household response rate for 2008 was $84.9 \%$.

\section{Item Nonresponse}

Item nonresponse for each of the sociodemographic indicators shown in this report generally was about $1 \%$ or less, with the exception of poverty status, which is based on detailed family income questions in the Family component of the questionnaire. Item nonresponse for the poverty indicator was $19.4 \%$. To minimize the possible bias that could be introduced from a relatively large amount of missing income data, NHIS-imputed income files were used (7). These data are produced by multiple imputation, a technique that allows the extra variation due to imputation to be accounted for in the data analysis. Item nonresponse rates were $0.2 \%$ for the health characteristics of dentition status, $1.2 \%$ for current smoking status, $2.3 \%$ for current drinking status, and $0.1 \%$ for diabetes status. For the oral health items, nonresponse rates ranged from $0.0 \%$ to $5.7 \%$. The denominators for the estimates shown in the tables exclude persons with unknown oral health related information for a given table. Results for persons with unknown sociodemographic characteristics are not shown separately, but are included in the totals for Tables 1-7.

\section{Estimation Procedures and Significance Testing}

All estimates and associated standard errors shown in this report were generated using SUDAAN (6), a software package designed to account for complex sample designs such as that of NHIS. All estimates were weighted using the Sample Adult record weight to represent the U.S. civilian noninstitutionalized population aged 18 and over.

\section{Tests of Significance}

Statistical tests performed to assess significance of differences in the estimates were two tailed with no adjustments for multiple comparisons. The test statistic used to determine significance of differences between two percentages was

$$
Z=\frac{\left|X_{a}-X_{b}\right|}{\sqrt{S_{a}^{2}+S_{b}^{2}}},
$$

where $X_{a}$ and $X_{b}$ are the two percentages being compared, and $S_{a}$ and $S_{b}$ are the SUDAAN-calculated standard errors of those percentages. The critical value used for two-sided tests at the 0.05 level was 1.96 .

\section{Relative Standard Error}

Estimates with a relative standard error of greater than $30 \%$ but less than or equal to $50 \%$ are identified with an asterisk and should be used with caution as they do not meet standards of reliability or precision. Estimates with a relative standard error of greater than $50 \%$ are indicated with a dagger $(\dagger)$ and are not shown. The relative standard errors are calculated as follows:

Relative standard error (as a percentage $)=(\mathrm{SE} / \mathrm{Est}) 100$, where $\mathrm{SE}$ is the standard error of the estimate, and Est is the estimate. 


\section{Appendix II. Definitions of Selected Terms}

\section{Sociodemographic Terms}

Education-The categories of education for adults are based on years of school completed or highest degree obtained for persons aged 25 and over. Only years completed in a school that advances a person toward an elementary or high school diploma, General Educational Development high school equivalency diploma, or college, university, or professional degree are included. Education in other schools or home schooling is counted only if the credits are accepted in a regular school system.

Hispanic or Latino originHispanic or Latino origin includes persons of Mexican, Puerto Rican, Cuban, Central and South American, or Spanish origin. Persons of Hispanic or Latino origin may be of any race.

Nativity- "U.S. born" is defined as having been born in one of the 50 states, the District of Columbia, Puerto Rico, or any of the other U.S. territories (e.g., American Samoa, Guam, U.S. Virgin Islands, and Northern Marianas). Persons born in a foreign country to parents who were U.S. citizens were also defined as U.S. born. Foreign-born persons are defined as people living in the United States who were not U.S. citizens at birth. All naturalized citizens, legal permanent residents, illegal aliens, and persons on long-term temporary visas (such as students or guest workers) also fall in this category.

Poverty status or percentage of poverty level-Based on family income, family size, number of children in the family, and, for families with two or fewer adults, on the age of the adults in the family. The poverty level is based on definitions originally developed by the U.S. Social Security Administration. These include a set of income thresholds defining the federal poverty level that vary by family size and composition. The thresholds are updated annually by the U.S. Census Bureau to reflect changes in the Consumer Price Index for all urban consumers (8). The poverty status of families or individuals is classified relative to their appropriate poverty threshold. Estimates by poverty status are based on both reported and imputed family income. Family income information was completely missing for $9.4 \%$ of persons, and family income information was only reported in broad categories for an additional $22.0 \%$ of persons in 2008. Therefore, imputed family income was used for $31.4 \%$ of persons in 2008 using the National Health Interview Survey (NHIS) imputed income files (7). Using the U.S. Census Bureau's poverty thresholds, "poor" persons are defined as those with family incomes below the poverty threshold. "Near poor" persons have family incomes of $100 \%$ to less than $200 \%$ of the poverty threshold, and "not poor" persons have family incomes that are $200 \%$ of the poverty threshold or greater.

Race-The 1997 Office of Management and Budget (OMB) federal guidelines (9) for reporting race require that persons of "single race" be distinguished from persons of "multiple race." "Single race" refers to persons who indicated only a single-race group. In this report, three categories are shown for single-race individuals (white, black or African American, and Asian). Other subcategories of single-race persons and multiple-race persons are not shown due to statistical unreliability as measured by the relative standard errors of the estimates.

The text in this report uses shorter versions of the OMB race terms for conciseness, and the tables use the complete terms. For example, the category "black, single race" in a table is referred to as "black" in the text.

Region-In the geographic classification of the U.S. population, states are grouped into four regions used by the U.S. Census Bureau:
Region

States included

Northeast Maine, Vermont, New Hampshire, Massachusetts, Connecticut, Rhode Island, New York, New Jersey, and Pennsylvania;

Midwest Ohio, Illinois, Indiana, Michigan, Wisconsin, Minnesota, Iowa, Missouri, North Dakota, South Dakota, Kansas, and Nebraska;

South Delaware, Maryland, District of Columbia, West Virginia, Virginia, Kentucky, Tennessee, North Carolina, South Carolina, Georgia, Florida, Alabama, Mississippi, Louisiana, Oklahoma, Arkansas, and Texas;

West Washington, Oregon, California, Nevada, New Mexico, Arizona, Idaho, Utah, Colorado, Montana, Wyoming, Alaska, and Hawaii.

\section{Health Insurance Terms}

Comprehensive plan-Refers to a health insurance plan or policy that covers a variety of medical services. These plans are designed to cover general medical expenses such as blood work or visits to a doctor's office and health catastrophes such as heart attacks and cancer.

Comprehensive plan with dental coverage-Refers to a private health insurance plan that also has dental coverage based on a positive response to the following question: "Does [Plan name] pay for any of the costs for dental care?"

Dental insurance status-The examination of dental insurance status was limited to those persons who had private health insurance. Federal and state assistance programs for dental care are limited. The Children's Health 
Insurance Program (CHIP) requires states to provide limited dental coverage for enrolled children up to age 19 years (10). The Medicaid program requires states to provide limited dental services for most Medicaid-eligible individuals under age 21 , but there is no assistance to provide dental services to individuals aged 21 and over (10); therefore, due to the limited or nonexistent public coverage for dental care, this report limits the analysis of dental insurance status to adults aged 18-64 who have private health insurance.

Health insurance coverage-NHIS respondents were asked about their health insurance coverage at the time of the interview. Respondents reported whether they were covered by private insurance (obtained through the employer or workplace, purchased directly, or through a local or community program), Medicare, Medigap (supplemental Medicare coverage), Medicaid, CHIP, Indian Health Service (IHS), military coverage (including VA, TRICARE, or CHAMP-VA), a state-sponsored health plan, another government program, or any single-service plans. This information was used to form a health insurance hierarchy that consisted of four mutually exclusive categories (11). Persons with more than one type of health insurance were assigned to the first appropriate category in the following hierarchy: private coverage, Medicaid (includes persons with Medicare, Medigap, Medicaid, CHIP, or a state-sponsored health plan), other coverage (includes any type of military coverage or another government program), and uninsured (also includes persons who are only covered by IHS or only have single-service plans).

Private health insurance-Indicated when respondents reported that they were covered by private health insurance through an employer, or union, or individual purchase. Private health insurance includes managed care such as health maintenance organizations and does not include military health plans.

Single-service dental plan-Refers to a private insurance plan that only pays for dental care.

\section{Oral Health Terms}

Comparative oral health status_-Based on the question: "Would you say the condition of your mouth and [if dentate] teeth is better than, the same as or not as good as other people your age?"

Dental visit-Refers to any visit to a dentist's office (including oral surgeons, orthodontists, or other dental specialists) for treatment or advice, including services by a technician or hygienist acting under a dentist's supervision.

Dentition status includes:

Edentulous persons-Refers to persons who have lost all of their permanent teeth. An edentulous person may have dentures.

Dentate persons-Refers to persons who have not lost all of their permanent teeth.

Interval since last dental visit-Refers to length of time prior to the week of interview since a dentist or dental hygienist was last visited for treatment or advice of any type.

Oral health status-Based on the question: "How would you describe the condition of your mouth and [if dentate] teeth? Would you say very good, good, fair or poor?"

Problems with mouth-Based on the question: "DURING THE PAST 6 MONTHS, have you had any of the following problems that lasted more than a day? .. Pain in your jaw joint, ... Sores in your mouth, ... Difficulty eating or chewing, ... Bad breath, . . Dry mouth."

Problems with teeth-Based on the question asked among dentate adults: "DURING THE PAST 6 MONTHS, have you had any of the following problems? ... A toothache or sensitive teeth, ... .Bleeding gums, ....Crooked teeth, ... Broken or missing teeth, ... .Stained or discolored teeth, ....Loose teeth not due to an injury, ... Broken or missing fillings."

Reason for not seeing a dentist or other dental health professional for a mouth or tooth problem-Based on the question: "DURING THE PAST 6
MONTHS, why didn't you see a dentist for the problems with your mouth or teeth? .. . You didn't think it was important, ... The problem went away, ...YYou could not afford treatment or you didn't have insurance, ... . You didn't have transportation,... .You were afraid to see a dentist, ... . You were waiting for an appointment, ... You didn't think a dentist could fix the problem."

Unmet dental need due to costBased on the question: "DURING THE PAST 12 MONTHS, was there any time when you needed any of the following but didn't get it because you couldn't afford it? ... Dental care (including check-ups)?"

\section{Health Behavior Terms}

\section{Current drinking status includes:}

Lifetime abstainer-Refers to adults who had fewer than 12 drinks in their entire lifetime.

Former drinker-Refers to adults who had 12 drinks or more in their lifetime, but had no drinks in the past year.

Current light or infrequent drinker-Refers to adults who had at least 12 drinks in their lifetime and 1-11 drinks in the past year (infrequent) or 3 drinks or fewer per week, on average (light).

Current moderate or heavier drinker-Refers to moderate drinkers as adults who had at least 12 drinks in their lifetime and more than 3 drinks per week up to 14 drinks per week (on average) for men and more than 3 drinks per week up to 7 drinks per week (on average) for women. Adults who had at least 12 drinks in their lifetime and more than 14 drinks per week (on average) for men and more than 7 drinks per week (on average) for women were heavier drinkers.

\section{Current smoking status includes:}

Current-Refers to adults who have smoked at least 100 cigarettes in their lifetime and currently smoke cigarettes every day or somedays. 
Former-Refers to adults who have smoked at least 100 cigarettes in their lifetime, but currently do not smoke at all.

Never-Refers to adults who never smoked a cigarette or who smoked fewer than 100 cigarettes in their entire lifetime. 


\section{Vital and Health Statistics Series Descriptions}

\section{ACTIVE SERIES}

Series 1. Programs and Collection Procedures-This type of report describes the data collection programs of the National Center for Health Statistics. Series 1 includes descriptions of the methods used to collect and process the data, definitions, and other material necessary for understanding the data.

Series 2. Data Evaluation and Methods Research-This type of report concerns statistical methods and includes analytical techniques, objective evaluations of reliability of collected data, and contributions to statistical theory. Also included are experimental tests of new survey methods, comparisons of U.S. methodologies with those of other countries, and as of 2009 , studies of cognition and survey measurement, and final reports of major committees concerning vital and health statistics measurement and methods.

Series 3. Analytical and Epidemiological Studies-This type of report presents analytical or interpretive studies based on vital and health statistics. As of 2009, Series 3 also includes studies based on surveys that are not part of continuing data systems of the National Center for Health Statistics and international vital and health statistics reports.

Series 10. Data From the National Health Interview Survey-This type of report contains statistics on illness; unintentional injuries; disability; use of hospital, medical, and other health services; and a wide range of special current health topics covering many aspects of health behaviors, health status, and health care utilization. Series 10 is based on data collected in this continuing national household interview survey.

Series 11. Data From the National Health Examination Survey, the National Health and Nutrition Examination Surveys, and the Hispanic Health and Nutrition Examination SurveyIn this type of report, data from direct examination, testing, and measurement on representative samples of the civilian noninstitutionalized population provide the basis for (1) medically defined total prevalence of specific diseases or conditions in the United States and the distributions of the population with respect to physical, physiological, and psychological characteristics, and (2) analyses of trends and relationships among various measurements and between survey periods.

Series 13. Data From the National Health Care Survey-This type of report contains statistics on health resources and the public's use of health care resources including ambulatory, hospital, and long-term care services based on data collected directly from health care providers and provider records.

Series 20. Data on Mortality-This type of report contains statistics on mortality that are not included in regular, annual, or monthly reports. Special analyses by cause of death, age, other demographic variables, and geographic and trend analyses are included.

Series 21. Data on Natality, Marriage, and Divorce-This type of report contains statistics on natality, marriage, and divorce that are not included in regular, annual, or monthly reports. Special analyses by health and demographic variables and geographic and trend analyses are included.

Series 23. Data From the National Survey of Family Growth-These reports contain statistics on factors that affect birth rates, including contraception and infertility; factors affecting the formation and dissolution of families, including cohabitation, marriage, divorce, and remarriage; and behavior related to the risk of HIV and other sexually transmitted diseases. These statistics are based on national surveys of women and men of childbearing age.

\section{DISCONTINUED SERIES}

Series 4. Documents and Committee Reports-These are final reports of major committees concerned with vital and health statistics and documents. The last Series 4 report was published in 2002. As of 2009, this type of report is included in Series 2 or another appropriate series, depending on the report topic.

Series 5. International Vital and Health Statistics Reports-This type of report compares U.S. vital and health statistics with those of other countries or presents other international data of relevance to the health statistics system of the United States. The last Series 5 report was published in 2003. As of 2009, this type of report is included in Series 3 or another series, depending on the report topic.

Series 6. Cognition and Survey Measurement-This type of report uses methods of cognitive science to design, evaluate, and test survey instruments. The last Series 6 report was published in 1999. As of 2009, this type of report is included in Series 2.

Series 12. Data From the Institutionalized Population SurveysThe last Series 12 report was published in 1974. Reports from these surveys are included in Series 13.

Series 14. Data on Health Resources: Manpower and FacilitiesThe last Series 14 report was published in 1989. Reports on health resources are included in Series 13.

Series 15. Data From Special Surveys-This type of report contains statistics on health and health-related topics collected in special surveys that are not part of the continuing data systems of the National Center for Health Statistics. The last Series 15 report was published in 2002. As of 2009, reports based on these surveys are included in Series 3.

Series 16. Compilations of Advance Data From Vital and Health Statistics-The last Series 16 report was published in 1996 All reports are available online, and so compilations of Advance Data reports are no longer needed.

Series 22. Data From the National Mortality and Natality SurveysThe last Series 22 report was published in 1973. Reports from these sample surveys, based on vital records, are published in Series 20 or 21 .

Series 24. Compilations of Data on Natality, Mortality, Marriage, and Divorce-The last Series 24 report was published in 1996. All reports are available online, and so compilations of reports are no longer needed.

For answers to questions about this report or for a list of reports published in these series, contact:

Information Dissemination Staff

National Center for Health Statistics

Centers for Disease Control and Prevention

3311 Toledo Road, Room 5412

Hyattsville, MD 20782

1-800-232-4636

E-mail: cdcinfo@cdc.gov

Internet: http://www.cdc.gov/nchs 


\section{U.S. DEPARTMENT OF}

\section{HEALTH \& HUMAN SERVICES}

MEDIA MAIL

Centers for Disease Control and Prevention

POSTAGE \& FEES PAID $\mathrm{CDC} / \mathrm{NCHS}$

National Center for Health Statistics

3311 Toledo Road

Hyattsville, MD 20782

PERMIT NO. G-284

OFFICIAL BUSINESS

PENALTY FOR PRIVATE USE, $\$ 300$ 